\title{
High-Temperature Ionization in Protoplanetary Disks
}

\author{
Steven J. Desch \\ School of Earth and Space Exploration, Arizona State University, PO Box 871404, Tempe, \\ AZ 85287-1404 \\ Neal J. Turner \\ Jet Propulsion Laboratory, Mail Stop 169-506, California Institute of Technology, 4800 Oak \\ Grove Drive, Pasadena, CA 91109
}

Received __; accepted _ 


\begin{abstract}
We calculate the abundances of electrons and ions in the hot ( $\gtrsim 500 \mathrm{~K})$, dusty parts of protoplanetary disks, treating for the first time the effects of thermionic and ion emission from the dust grains. High-temperature ionization modeling has involved simply assuming that alkali elements such as potassium occur as gasphase atoms and are collisionally ionized following the Saha equation. We show that the Saha equation often does not hold, because free charges are produced by thermionic and ion emission and destroyed when they stick to grain surfaces. This means the ionization state depends not on the first ionization potential of the alkali atoms, but rather on the grains' work functions. The charged species' abundances typically rise abruptly above about $800 \mathrm{~K}$, with little qualitative dependence on the work function, gas density, or dust-to-gas mass ratio. Applying our results, we find that protoplanetary disks' dead zone, where high diffusivities stifle magnetorotational turbulence, has its inner edge located where the temperature exceeds a threshold value $\approx 1000 \mathrm{~K}$. The threshold is set by ambipolar diffusion except at the highest densities, where it is set by Ohmic resistivity. We find that the disk gas can be diffusively loaded onto the stellar magnetosphere at temperatures below a similar threshold. We investigate whether the "shortcircuit" instability of current sheets can operate in disks and find that it cannot, or works only in a narrow range of conditions; it appears not to be the chondrule formation mechanism. We also suggest that thermionic emission is important for determining the rate of Ohmic heating in hot Jupiters.
\end{abstract}

Subject headings: accretion, accretion disks, planetary systems: protoplanetary disks, solar system: formation 


\section{Introduction}

Protoplanetary disks' evolution is governed by the extraction of orbital angular momentum needed for accretion to proceed. Magnetic forces are among the prime candidates to drive the extraction. Modeling the disks therefore requires knowing how readily the magnetic fields diffuse through the material. Hence the ionization state of the gas is key.

In particular, the magnetorotational instability (MRI) is suppressed unless the electron fraction is $x_{\mathrm{e}}=n_{\mathrm{e}} / n_{\mathrm{H} 2} \gtrsim 10^{-13}$ (Jin 1996; Gammie 1996), where $n_{\mathrm{H} 2}$ is the number density of $\mathrm{H}_{2}$ molecules. Launching disk winds requires $x_{\mathrm{e}} \gtrsim 10^{-11}-10^{-10}$ (Bai \& Stone 2013). The gas ionization likewise bears on the existence of lightning (Desch \& Cuzzi 2000) and current sheets (McNally et al. 2013), two proposed mechanisms for transiently heating and melting chondrules (millimeter-sized inclusions in chondritic meteorites) in the solar nebula. Assessing the extent of the MRI and the heating of chondrules requires knowing the ionization rates, especially in the disks' denser midplane regions.

Ionization in the cooler parts of disks, where temperatures $T \lesssim 500 \mathrm{~K}$, has been studied in detail. Several non-thermal processes are important at these low temperatures, with ionization rates per volume often expressed as $\zeta n_{\mathrm{H} 2}$. Far-ultraviolet (FUV) photons from the central star can ionize gas at a rate $\zeta_{\mathrm{UV}} \sim 10^{-8} \mathrm{~s}^{-1}$ at $1 \mathrm{AU}$, but are attenuated by column densities $\lesssim 0.1 \mathrm{~g} \mathrm{~cm}^{-3}$ (Perez-Becker \& Chiang 2011). Similarly, X-rays from the star can ionize at rates $\zeta_{\mathrm{x}} \sim 10^{-11} \mathrm{~s}^{-1}$ at $1 \mathrm{AU}$, but are attenuated by column densities $\lesssim 10 \mathrm{~g} \mathrm{~cm}^{-3}$ (Glassgold et al. 1997; Igea \& Glassgold 1999; Ercolano \& Glassgold 2013). Galactic cosmic rays, if not shielded by stellar winds (Cleeves et al. 2013a), can ionize at

rates $\zeta_{\mathrm{GCR}} \sim 10^{-17} \mathrm{~s}^{-1}$, but are attenuated by column densities $\sim 100 \mathrm{~g} \mathrm{~cm}^{-3}$ (Umebayashi \& Nakano 1981). In the innermost few AU of a protoplanetary disk, the expected column densities are $\gg 10^{3} \mathrm{~g} \mathrm{~cm}^{-2}$, so that the dominant non-thermal ionization sources are $\beta$ 
particles from radioactive decays of species like ${ }^{40} \mathrm{~K}$, leading to $\zeta_{\mathrm{rad}} \sim 10^{-21} \mathrm{~s}^{-1}$ (Umebayashi \& Nakano 1981); or ${ }^{26} \mathrm{Al}$ (if present), leading to $\zeta_{\text {rad }} \lesssim 10^{-18} \mathrm{~s}^{-1}$ (Cleeves et al. 2013b). In the disks' cold midplane regions, this is the expected ionization rate, and it is quite low.

In chemical equilibrium in the dense midplane gas, the electron density is found by balancing the ionization rate against adsorption, and subsequent recombination, of electrons on grain surfaces. Assuming monodisperse grains with radius $a_{\mathrm{gr}}=1 \mu \mathrm{m}$, internal density $\rho_{\mathrm{s}}=3 \mathrm{~g} \mathrm{~cm}^{-3}$, and dust-to-gas mass ratio $\rho_{\mathrm{gr}} / \rho_{\mathrm{g}}=0.01$, the equation describing this balance is

$$
\zeta n_{\mathrm{H}_{2}}=n_{\mathrm{gr}} \pi a_{\mathrm{gr}}^{2} n_{\mathrm{e}}\left(\frac{8 k T}{\pi m_{\mathrm{e}}}\right)^{1 / 2} S_{\mathrm{e}}
$$

(Sano et al. 2000), where $n_{\mathrm{gr}}=\left(\rho_{\mathrm{gr}} / \rho_{\mathrm{g}}\right)\left(1.4 n_{\mathrm{H}_{2}} m_{\mathrm{H}_{2}}\right) /\left(4 \pi \rho_{\mathrm{s}} a_{\mathrm{gr}}^{3} / 3\right)$ is the number density of grains, $S_{\mathrm{e}}$ the sticking coefficient of electrons colliding with grains, and other variables have their usual meanings. From this we find $n_{\mathrm{e}} \sim 10^{-3} \mathrm{~cm}^{-3}$ (assuming $\zeta=10^{-18} \mathrm{~s}^{-1}, T=300 \mathrm{~K}$ and $\left.S_{\mathrm{e}}=0.6\right)$. For a typical midplane gas density inside a few $\mathrm{AU}, n_{\mathrm{H}_{2}} \gtrsim 10^{14} \mathrm{~cm}^{-3}$ (Weidenschilling 1977), $x_{\mathrm{e}} \lesssim 10^{-17}$, a value too low to be of significance to the MRI. Recognition of this fact led Gammie (1996) to suggest that protoplanetary disks' interiors, especially the parts within a few AU of their stars, are "dead zones" where the MRI cannot act.

Jin (1996) and Gammie (1996) were the first to recognize that very close to the central star, protoplanetary disks may be hot enough to sustain thermal ionizations, recoupling the magnetic field to the gas. Specifically, they pointed out that collisional ionization of gas-phase potassium atoms at temperatures $\gtrsim 1000 \mathrm{~K}$ would generate free electrons and ions. The threshold temperature of $1000 \mathrm{~K}$ is based on calculating the abundances of $\mathrm{K}^{+}$ ions and electrons using the Saha equation:

$$
\frac{n_{\mathrm{K}^{+}}}{n_{\mathrm{K}^{0}}}=\frac{g_{+}}{g_{0}} \frac{2}{n_{\mathrm{e}}}\left(\frac{2 \pi m_{\mathrm{e}} k T}{h^{2}}\right)^{3 / 2} \exp \left(-\frac{\mathrm{IP}}{k T}\right) .
$$

Here $g_{+}=1$ and $g_{0}=2$ are the statistical weights of the (ground states) of $\mathrm{K}^{+}$and $\mathrm{K}^{0}, k$ 
the Boltzmann constant, $h$ Planck's constant, $m_{\mathrm{e}}$ the electron mass, and IP $=4.34 \mathrm{eV}$ the first ionization potential energy of potassium. Assuming $n_{\mathrm{K}^{+}}=n_{\mathrm{e}}$ and that the abundance of $\mathrm{K}$ relative to $\mathrm{H}_{2}$ in a solar-composition gas is $x_{\mathrm{K}}=3.0 \times 10^{-7}$ (Lodders 2003), half of the $\mathrm{K}$ atoms are ionized when $T=1715 \mathrm{~K}$, for a density $n_{\mathrm{H}_{2}}=10^{14} \mathrm{~cm}^{-3}$ likely to occur near the midplane. More significantly for the MRI, $x_{\mathrm{e}}=10^{-13}$ when $T=865 \mathrm{~K}$, meaning substantial ionization would be expected if potassium were in the gas phase at this temperature. According to Lodders (2003), the 50\% condensation temperature of potassium is $1006 \mathrm{~K}$ (at $P=10^{-4} \mathrm{bar}$, or $n_{\mathrm{H}_{2}}=6 \times 10^{14} \mathrm{~cm}^{-3}$; the pressure dependence is weak). So it is fairer to say that above $1000 \mathrm{~K}$ the Saha equation predicts significant thermal ionization of potassium, and for temperatures between about 850 and $1000 \mathrm{~K}$, thermal ionization may or may not lead to significant ionization.

Other species contribute less strongly to thermal ionization than potassium. Because of the exponential dependence on the first ionization potential (IP), one might expect other alkali atoms to contribute to thermal ionization. The first ionization potentials of $\mathrm{Li}, \mathrm{Na}, \mathrm{K}$, Rb, and Cs are, respectively, 5.39, 5.14, 4.34, 4.18, and $3.89 \mathrm{eV}$. These elements' abundances relative to $\mathrm{H}_{2}$ are, respectively, $4.57 \times 10^{-9}, 4.73 \times 10^{-6}, 3.04 \times 10^{-7}, 5.51 \times 10^{-10}$, and $3.02 \times 10^{-11}$ (Lodders 2003). The first ionization potentials (FIP) and cosmochemical abundances of many elements are depicted in Figure 1. Repeating the analysis above, the temperature at which each alkali element contributes enough electrons to yield $x_{\mathrm{e}}=10^{-13}$ (at $n_{\mathrm{H}_{2}}=10^{14} \mathrm{~cm}^{-3}$ ) is $1149,975,865,932$, and $852 \mathrm{~K}$, respectively. Considering that cesium is in the gas phase above $800 \mathrm{~K}$ whereas potassium is not (Lodders 2003), one might expect Cs to be the dominant contributor of ions and electrons at temperatures between 850 and $1000 \mathrm{~K}$; however, the Cs is $10^{4}$ times less abundant, so that even if all Cs were ionized and only $0.1 \%$ of all $\mathrm{K}$ atoms were in the gas phase, potassium would still dominate over cesium. Thermal ionization of potassium is likely to be more important than that of other alkalis, and potassium is in any case an excellent proxy for other alkalis. Throughout 
this paper we will consider the case of potassium to represent the contributions of all the alkali elements to thermal ionization. 


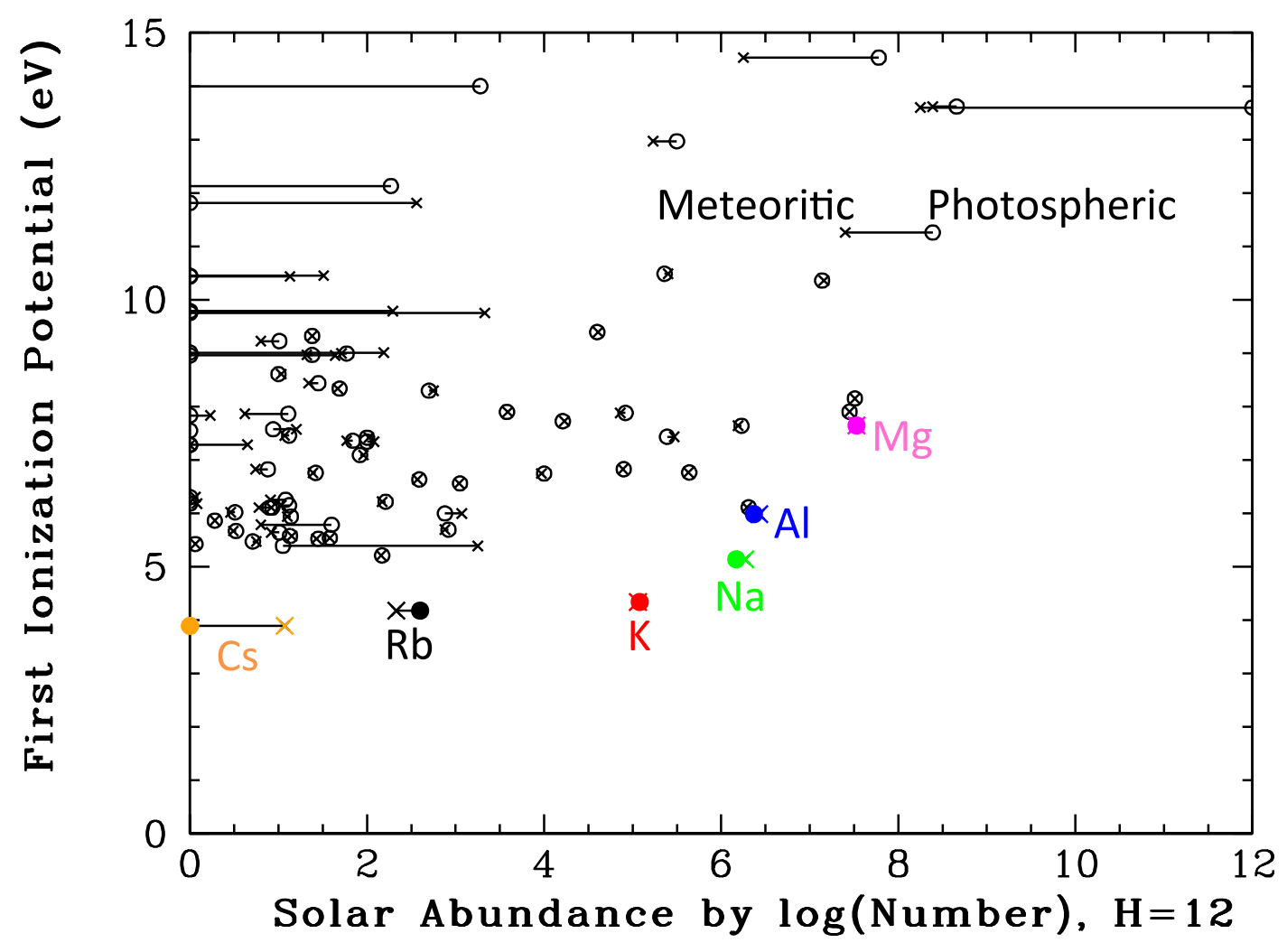

Fig. 1.- Cosmochemical abundances of various elements and their first ionization potentials (FIPs). Abundances (on a scale where the number of $\mathrm{H}$ atoms is $10^{12}$ ) are derived by Asplund et al. (2005) from analyses of carbonaceous chondrites ("meteoritic") or from spectrometry of the Sun ("photospheric"). Producing free electrons by thermal ionization requires the presence of elements with high abundance and low FIP, i.e., those elements toward the plot's lower right corner. The exponential sensitivity of the ionization fraction to FIP means potassium $(\mathrm{K})$ is most favored to provide free electrons by thermal ionization, with $\mathrm{Na}, \mathrm{Rb}$, and Cs potentially also important - if these elements occur in the gas phase rather than locked up in minerals. 
A criticism of this thermal ionization picture is that the Saha equation is valid only in local thermodynamic equilibrium, or LTE (Desch 1998). It relies on the collisional ionization of the gas-phase $\mathrm{K}$ atoms, e.g., $\mathrm{H}_{2}+\mathrm{K}^{0} \rightarrow \mathrm{H}_{2}+\mathrm{K}^{+}+\mathrm{e}^{-}$, being in detailed balance with its inverse reaction, three-body gas-phase recombination, $\mathrm{H}_{2}+\mathrm{K}^{+}+\mathrm{e}^{-} \rightarrow \mathrm{H}_{2}+\mathrm{K}^{0}$. But under conditions typical for protoplanetary disks, electrons are more likely to recombine with ions on the surfaces of grains. Desch (1998) argued that the electron density must be set by balancing collisional ionizations against collisions of electrons with dust grains. Once adsorbed on the grain surfaces, electrons and ions quantum tunnel from one adsorption site to the next, wandering across the surface until they find each other and recombine. The resulting neutral K atoms can then escape the grain. Dust grain surface-catalyzed recombinations are much faster than gas-phase recombinations, as the frequency of colliding with dust grains is $\sim n_{\mathrm{gr}} \pi a_{\mathrm{gr}}^{2}\left(8 k T / \pi m_{\mathrm{e}}\right)^{1 / 2} S_{\mathrm{e}} \sim 0.1 \mathrm{~s}^{-1}$ (at $T=1000 \mathrm{~K}$ and $n_{\mathrm{H}_{2}}=10^{14} \mathrm{~cm}^{-3}$, and assuming grains with radius $1 \mu \mathrm{m}$ and a dust-to-gas mass ratio 0.01$)$, whereas the frequency of recombining with an ion is $\sim k_{-2} n_{\mathrm{H}_{2}} n_{\mathrm{K}^{+}} \lesssim 10^{-11} \mathrm{~s}^{-1}$, where $k_{-2}$ is the recombination coefficient (see below), and we have assumed the ion density matches the electron density, and $x_{\mathrm{e}}=10^{-11}$. Not until $x_{\mathrm{e}} \gtrsim 10^{-3}$ do gas-phase recombinations dominate over grain-catalyzed recombinations. The dominance of grain effects invalidates the use of the Saha equation for potassium as a means of calculating the electron density.

The dominance of grain processes also points to an additional ionization source, not previously considered in the context of protoplanetary disks. The process that is the inverse of electrons and ions colliding with grains, resulting in neutral atoms leaving the grain, is the emission of electrons or ions following collisions of neutral atoms with grains. These processes are more commonly called thermionic emission and ion emission. When solids are heated to temperatures $\gtrsim 500 \mathrm{~K}$, they emit electrons and/or ions. The emission of electrons depends on the work function of the solids comprising the grains, which also affects whether evaporating atoms such as potassium are emitted as neutral atoms or ions. Including the 
thermionic and ion emission completely changes the role of dust grains in the ionization of the gas: instead of mopping up free charges, they generate them.

In this paper we examine the effects of thermionic and ion emission on the ionization state of high-temperature, dusty gas. In $\S 2$ we discuss the microphysical mechanisms of thermionic and ion emission, as well as the evaporation and condensation of $\mathrm{K}$ atoms, and provide quantitative rates and likely input parameters. In $\S 3$ we create a simple model treating evaporation and condensation of $\mathrm{K}$ atoms and ions, including ion emission and thermionic emission. We show that, because of the high work function of silicate grains, ion emission creates signficant ionization at temperatures around $800 \mathrm{~K}$, possibly as low as $600 \mathrm{~K}$. In $\S 4$ we discuss the impact on several aspects of protoplanetary disks. In particular, we address where the dead zone's inner edge should lie, what temperatures are needed for disk gas to diffuse into the star's magnetosphere, and whether including this new ionization source can enable the "short-circuit instability" of McNally et al. (2013). Thermionic and ion emission profoundly affect the ionization state of high-temperature gas in disks.

\section{High-Temperature Chemical Network}

\subsection{High-Temperature Ionization Effects}

In the dusty gas of protoplanetary disks, we cannot always use the Saha equation, and instead must check the rates of various ionization and recombination processes. What's more, protoplanetary disks are marked by transient temperature changes, and we are interested in how the ionization responds to the changing conditions. Thus motivated, in this section we lay out several processes' kinetic rates and construct a chemical network to find the steady-state abundances of electrons and ions as well as the timescales for the ionization chemistry to reach equilibrium. 
Our chemical network includes five species: electrons, molecular or atomic ions, $\mathrm{K}$ atoms bound to dust grains, gas-phase neutral $\mathrm{K}$ atoms, and gas-phase $\mathrm{K}$ ions. Energetic particles (cosmic rays, radioactive decay products, etc.), and at high temperatures also gas-phase reactions, ionize the gas molecules; ions and electrons recombine in the gas phase. We also include dust grains, calculating their steady-state charge. All the other species can collide with and condense on or chemically react with the grains. The grains can lose $\mathrm{K}$ atoms at high temperatures, and can eject electrons and ions by the thermionic effect and by ion emission, respectively. Before discussing the chemical network, we review these chemical processes.

\subsection{Gas-Phase Collisional Ionization and Recombination}

As discussed above, the $\mathrm{H}_{2}$ molecules' ionization can come from energetic particles such as Galactic cosmic rays, X-ray photons, or particles from radioactive decays. The number of ionizations per volume per time is defined as $\zeta n_{\mathrm{H}_{2}}$. We are most interested in protoplanetary disks' interiors, so we assume that the cosmic rays and X-rays are shielded, leaving only ionization by the decay of radioactive elements. If short-lived radionuclides are not present, then decay of ${ }^{40} \mathrm{~K}$, as well as ${ }^{238} \mathrm{U},{ }^{235} \mathrm{U}$ and ${ }^{232} \mathrm{Th}$, dominate, yielding $\zeta \approx 6.9 \times 10^{-22} \mathrm{~s}^{-1}$ (Umebayashi \& Nakano 1981). If the short-lived $\left(t_{1 / 2}=0.71 \mathrm{Myr}\right)$ radionuclide ${ }^{26} \mathrm{Al}$ is present at levels inferred from meteorites (MacPherson et al. 1995), then $\zeta \sim 10^{-18} \mathrm{~s}^{-1}$ (Umebayashi \& Nakano 2009). Our focus here is on high-temperature ionization, so we arbitrarily take $\zeta=6.9 \times 10^{-22} \mathrm{~s}^{-1}$ in what follows. (If ionizations by ${ }^{26} \mathrm{Al}$ are significant, the ion and electron densities would rise sharply above $800 \mathrm{~K}$ instead of $700 \mathrm{~K}$.) The $\mathrm{H}_{2}^{+}$ions quickly exchange charge with other molecules to produce ions such as $\mathrm{HCO}^{+}$, which then also exchange charge with free atoms, creating ions such as $\mathrm{Mg}^{+}$. We assume these steps are faster than the other reactions, so each ionization of $\mathrm{H}_{2}$ quickly 
yields an atomic ion. We do not consider $\mathrm{K}^{+}$as one of these ions, because other species like $\mathrm{Mg}$ are more abundant. Instead we assume $\mathrm{K}^{+}$ions are produced mainly by thermal ionization.

Atomic ions and free electrons can recombine in the gas phase. We assume a rate per volume $\beta n_{\mathrm{e}} n_{\mathrm{i}}$, where $\beta=3 \times 10^{-11} T^{-1 / 2} \mathrm{~cm}^{3} \mathrm{~s}^{-1}, T$ measured in $\mathrm{K}$ (Oppenheimer \& Dalgarno 1974). Ions and free electrons can also be adsorbed onto grain surfaces, at rates

$$
\mathcal{R}_{\mathrm{i}, \text { ads }}=n_{\mathrm{i}} n_{\mathrm{gr}} \pi a_{\mathrm{gr}}^{2}\left(8 k T / \pi m_{\mathrm{i}}\right)^{1 / 2} S_{\mathrm{i}} \tilde{J}_{\mathrm{i}} \equiv n_{\mathrm{i}} \nu_{\mathrm{i}, \mathrm{ads}}
$$

and

$$
\mathcal{R}_{\mathrm{e}, \text { ads }}=n_{\mathrm{e}} n_{\mathrm{gr}} \pi a_{\mathrm{gr}}^{2}\left(8 k T / \pi m_{\mathrm{e}}\right)^{1 / 2} S_{\mathrm{i}} \tilde{J}_{\mathrm{e}} \equiv n_{\mathrm{e}} \nu_{\mathrm{e}, \mathrm{ads}},
$$

where $\tilde{J}_{\mathrm{i}}$ and $\tilde{J}_{\mathrm{e}}$ reflect modifications to the collision cross section due to the grain charge (Draine \& Sutin 1987).

Thermal ionization of alkali atoms occurs if they are in the gas phase and suffer collisions with ambient molecules, mostly $\mathrm{H}_{2}$. This process can be represented by the reaction

$$
\mathrm{H}_{2}+\mathrm{K}^{0} \rightarrow \mathrm{H}_{2}+\mathrm{K}^{+}+\mathrm{e}^{-}
$$

In the gas phase, electrons and ions can radiatively recombine, as follows,

$$
\mathrm{K}^{+}+\mathrm{e}^{-} \rightarrow \mathrm{K}^{0}+\gamma
$$

but in the regions of protoplanetary disks where temperatures are $\gtrsim 500 \mathrm{~K}$ (the midplane regions well inside $1 \mathrm{AU}$ ), the gas densities are likely high enough that three-body recombinations dominate:

$$
\mathrm{H}_{2}+\mathrm{K}^{+}+\mathrm{e}^{-} \rightarrow \mathrm{H}_{2}+\mathrm{K}^{0}
$$

We justify this statement below. 
Literature on the collisional ionization and recombination rates is sparse. Pneumann \& Mitchell (1965) present a kinetic rate, but cite no measurements or sources. Their rate also appears to conflict with that provided by Ashton \& Hayhurst (1973). Since the latter is experimentally determined by analyzing the chemical reactions in flames, it is our preferred value. Following Ashton \& Hayhurst (1973), collisional ionization takes place at a rate per unit volume

$$
\mathcal{R}_{\text {gas,ion }}=k_{2} n_{\mathrm{H}_{2}} n_{\mathrm{K}^{0}}
$$

where

$$
k_{2}=(9.9 \pm 2.7) \times 10^{-9} T^{1 / 2} \exp \left(-\frac{\mathrm{IP}}{k T}\right) \mathrm{cm}^{3} \mathrm{~s}^{-1}
$$

and $T$ is measured in $\mathrm{K}$. Here $\mathrm{IP}=4.34 \mathrm{eV}$ for $\mathrm{K}$, and $\mathrm{IP} / k=50350 \mathrm{~K}$. The 3-body gas-phase recombination rate is

$$
\mathcal{R}_{\text {gas,rec }}=k_{-2} n_{\mathrm{H}_{2}} n_{\mathrm{K}}+n_{\mathrm{e}}
$$

where

$$
k_{-2}=(4.4 \pm 1.1) \times 10^{-24} T^{-1} \mathrm{~cm}^{6} \mathrm{~s}^{-1}
$$

and again $T$ is measured in Kelvin (Ashton \& Hayhurst 1973).

The two coefficients $k_{2}$ and $k_{-2}$ must be related to each other. In the absence of dust, these would be the only rates affecting the ionization fraction, and should be in detailed balance with each other in local thermodynamic equilibrium. The ionization fractions found using these coefficients thus should conform to the Saha equation. Hence, $\mathcal{R}_{\text {gas,rec }}=\mathcal{R}_{\text {gas,ion }}$, and $n_{\mathrm{K}^{+}} n_{\mathrm{e}} k_{-2}=n_{\mathrm{K}^{0}} k_{2}$, and therefore we expect

$$
\frac{k_{2}}{k_{-2}}=\frac{n_{\mathrm{K}^{+}} n_{\mathrm{e}}}{n_{\mathrm{K}_{0}}}=\frac{2 g_{+}}{g_{0}}\left(\frac{2 \pi m_{\mathrm{e}} k T}{h^{2}}\right)^{3 / 2} \exp \left(-\frac{\mathrm{IP}}{k T}\right)=2.415 \times 10^{15} T^{3 / 2} \exp \left(-\frac{\mathrm{IP}}{k T}\right)
$$

The values from Ashton \& Hayhurst (1973) yield

$$
\frac{k_{2}}{k_{-2}}=(2.3 \pm 0.8) \times 10^{15} T^{3 / 2} \exp \left(-\frac{\mathrm{IP}}{k T}\right)
$$


consistent with the Saha equation.

Recombinations by 3 -body reactions proceed at a rate $\left(k_{-2} n_{\mathrm{H}_{2}}\right) n_{\mathrm{K}^{+}} n_{\mathrm{e}}$, which is to be compared to the rate of radiative 2 -body recombinations, $\beta n_{\mathrm{K}}+n_{\mathrm{e}}$. For typical radiative recombinations, $\beta \sim 3 \times 10^{-11} T^{-1 / 2} \mathrm{~cm}^{3} \mathrm{~s}^{-1}$ (Oppenheimer \& Dalgarno 1974). At $T=500 \mathrm{~K}, k_{-2} \approx 10^{-26} \mathrm{~cm}^{6} \mathrm{~s}^{-1}$, and if $n_{\mathrm{H}_{2}}=10^{14} \mathrm{~cm}^{-3}$, then $k_{-2} n_{\mathrm{H}_{2}} \sim 10^{-12} \mathrm{~cm}^{3} \mathrm{~s}^{-1}$. For

densities $n_{\mathrm{H}_{2}} \gtrsim 10^{14} \mathrm{~cm}^{-3}$, radiative recombinations of $\mathrm{K}^{+}$and electrons are less significant than 3-body recombinations, but we include both rates in our calculations.

\subsection{Thermionic Emission}

Thermionic emission, the ejection of electrons from heated solids, is a long recognized effect and the basis for the cathode ray tube. The rate at which electrons are emitted, per surface area of solid, is described by Richardson's law,

$$
j(T)=\lambda_{\mathrm{R}} \frac{4 \pi m_{\mathrm{e}}(k T)^{2}}{h^{3}} \exp \left(-\frac{W}{k T}\right),
$$

where $\lambda_{\mathrm{R}}$ is a dimensionless number of order unity depending on the solid material, and is typically $\approx 1 / 2$ (Crowell et al. 1965).

Thermionic emission is sensitive to $W$, the work function of the material - that is, the energy needed to make an electron escape the solid. As the grains lose electrons and develop a positive charge $+Z e$, removing more electrons requires extra work to overcome the Coulomb force. The effective work function is

$$
W_{\text {eff }}=W+\frac{Z e^{2}}{a_{\text {gr }}} .
$$

For conductors, the escape takes place from the top of the conduction band, and the work function is easily measured and commonly tabulated. For example, the work function of graphite is $4.62 \mathrm{eV}$ (Jain \& Krishnan 1952), Fe has a work function of $4.31 \mathrm{eV}$, and that of $\mathrm{Ni}$ is $4.50 \mathrm{eV}$ (Fomenko 1966). 
Unfortunately, the grains likely to occur in the solar nebula and other protoplanetary disks contain many insulating materials, making determining the work function difficult. The best analogs to the grains in the solar nebula, and presumably in other protoplanetary disks as well, are the unaltered (pre-accretionary) matrix grains in chondrites. These grains are composed of crystalline, Mg-rich olivines $\left[\mathrm{Mg}_{2} \mathrm{SiO}_{4}\right]$ and pyroxenes $\left[\mathrm{MgSiO}_{3}\right]$; relatively Fe-rich amorphous silicates $\left[(\mathrm{Mg}, \mathrm{Fe})_{2} \mathrm{SiO}_{4}\right]$ and $\left[(\mathrm{Mg}, \mathrm{Fe}) \mathrm{SiO}_{3}\right]$; and a smaller fraction (a few percent) of grains of metallic Fe,Ni and their sulfides (Scott \& Krot 2005). Metallic FeNi grains are conducting, with a work function presumably $\approx 4.4 \mathrm{eV}$, but the silicates are insulators. Because of this, work functions of silicates are difficult to measure, and few data exist in the literature. Quartz $\left(\mathrm{SiO}_{2}\right)$ has a work function $5.0 \mathrm{eV}$, and mica (a phyllosilicate) has a work function $4.8 \mathrm{eV}$ (Fomenko 1966), but the authors were unable to find any other direct measurements for specific silicate minerals. Far better than quartz and mica as analogs for solar nebula dust, however, is the JSC-1 lunar regolith simulant (McKay et al. 1993), composed of a mixture of $40 \mathrm{wt} \%$ ferromagnesian olivine $\left[(\mathrm{Fe}, \mathrm{Mg})_{2} \mathrm{SiO}_{4}\right]$, 40 wt\% plagioclase $\left[\mathrm{NaAlSi}_{3} \mathrm{O}_{8}\right.$ and $\left.\mathrm{CaAl}_{2} \mathrm{Si}_{2} \mathrm{O}_{8}\right]$, the remainder comprised of Fe- and Ti-rich minerals like ilmenite $\left(\mathrm{FeTiO}_{3}\right)$, anatase $\left[\mathrm{TiO}_{2}\right]$, magnetite $\left[\mathrm{Fe}_{3} \mathrm{O}_{4}\right]$, hematite $\left[\mathrm{Fe}_{2} \mathrm{O}_{3}\right]$ and pseudobrookite $\left[\mathrm{Fe}_{2} \mathrm{TiO}_{5}\right]$. Trigwell et al. (2009) examined how JSC-1 simulant is charged when brought into contact with different substances, and found it was negatively charged by $\mathrm{Al}$ (work function $4.28 \mathrm{eV}), \mathrm{Cu}(4.65 \mathrm{eV})$ and stainless steel $(5.04 \mathrm{eV})$, but positively charged by the organic polymer PTFE (work function $5.75 \mathrm{eV}$ ). The acquired charge correlates with the work function of the other material, and the correlation strongly suggests JSC-1 itself has an effective work function somewhere between 5.0 and $5.4 \mathrm{eV}$. Feuerbacher et al. (1972) examined the photoelectric effect using JSC-1 simulant and found the threshold for photoelectron emission implies an effective work function close to $5.0 \mathrm{eV}$. Based on these measurements, we adopt a work function of $5.0 \mathrm{eV}$ for solar nebula dust grains. 
With the work function defined, the rate of electron production per volume due to thermionic emission from dust is

$$
\mathcal{R}_{\text {therm }}=n_{\text {gr }} 4 \pi a_{\text {gr }}^{2} j(T)=n_{\text {gr }} 4 \pi a_{\text {gr }}^{2} \lambda_{\mathrm{R}} \frac{4 \pi m_{\mathrm{e}}(k T)^{2}}{h^{3}} \exp \left(-\frac{W_{\text {eff }}}{k T}\right) .
$$

Again, for silicates we adopt $W=5.0 \mathrm{eV}$. Since data are lacking for the coefficient $\lambda_{\mathrm{R}}$, we adopt $\lambda_{\mathrm{R}}=1 / 2$.

The inverse of thermionic emission is electrons sticking on dust grains, which occurs at a rate $n_{\mathrm{e}} \nu_{\text {ads }}$. In the absence of gas-phase processes affecting electrons, thermionic emission and its inverse could be expected to be in detailed balance, with $\mathcal{R}_{\text {therm }}=\mathcal{R}_{\mathrm{e}, \text { ads }}$ and

$$
\lambda_{\mathrm{R}} \frac{4 \pi m_{\mathrm{e}} k^{2} T^{2}}{h^{3}} \exp \left(-\frac{W_{\mathrm{eff}}}{k T}\right)=\tilde{J}_{\mathrm{e}} S_{\mathrm{e}} n_{\mathrm{e}} \frac{1}{4}\left(\frac{8 k T}{\pi m_{\mathrm{e}}}\right)^{1 / 2}
$$

Note that since the electrons' production and destruction rates both scale with the grain surface area, the grain density and radii drop out of the balance equation. Solving for $n_{\mathrm{e}}$ yields

$$
n_{\mathrm{e}}=\frac{4 \lambda_{\mathrm{R}}}{2 \tilde{J}_{\mathrm{e}} S_{\mathrm{e}}}\left(\frac{2 \pi m_{\mathrm{e}} k T}{h^{2}}\right)^{3 / 2} \exp \left(-\frac{W_{\mathrm{eff}}}{k T}\right) .
$$

This is identical in form to a Saha equation, involving a charged and neutral species $X^{+}$ and $X^{0}$ with statistical weights $g^{+}$and $g^{0}$, provided $\left(n_{\mathrm{X}^{0}} / n_{\mathrm{X}^{+}}\right)\left(g_{+} / g_{0}\right)=\lambda_{\mathrm{R}} /\left(2 \tilde{J}_{\mathrm{e}} S_{\mathrm{e}}\right) \sim 1$. Potassium ion and electron densities essentially follow a Saha equation, but with an energy equal to $W_{\text {eff }}$, which in practice is typically in the range of 3 to $4 \mathrm{eV}$ (see below), not the ionization potential IP $=4.34 \mathrm{eV}$. In this way the dust grains fundamentally alter the gas ionization state, even in the high-temperature limit, where their effects are described by a Saha-like equation.

\subsection{Evaporation of Neutral and Ionized Potassium Atoms}

In addition to electrons, atoms can also emit ions. For the reasons outlined above, most relevant are $\mathrm{K}^{+}$ions. At high temperatures, potassium exists mostly in the gas phase, with 
$\mathrm{K}$ atoms that meet dust grains quickly vaporizing because of thermal vibrations. A single $\mathrm{K}$ atom bound to a grain surface vibrates on the lattice with a frequency $\nu$. We adopt a typical value $\nu=3.7 \times 10^{13} \mathrm{~s}^{-1}$, appropriate for $\mathrm{K}$ bound to Pt (Hagstrom et al. 2000). In any vibration cycle, there is a probability of vaporization $\exp \left(-E_{\mathrm{a}} / k T\right)$, where $E_{\mathrm{a}}$ is the activation energy, equal to the binding energy, typically several $\mathrm{eV}$. Defining $n_{\mathrm{K} \text {,cond }}$ as the number of $\mathrm{K}$ atoms in the grain minerals per volume of nebula, the vaporization rate per volume is

$$
\mathcal{R}_{\mathrm{K}, \text { evap }}=n_{\mathrm{K}, \text { cond }} \nu \exp \left(-\frac{E_{\mathrm{a}}}{k T}\right) \equiv n_{\mathrm{K}, \text { cond }} \nu_{\text {evap }}
$$

Balancing these vaporizations is the condensation of $\mathrm{K}$ atoms onto the grain surfaces. The total rate at which $\mathrm{K}$ atoms collide with dust grains is $\mathcal{R}_{\mathrm{K} 0 \text {,coll }}+\mathcal{R}_{\mathrm{K}+\text {,coll }}$, where

$$
\mathcal{R}_{\mathrm{K}+\text {, coll }}=n_{\mathrm{K}^{+}} n_{\mathrm{gr}} \pi a_{\mathrm{gr}}^{2}\left(\frac{8 k T}{\pi m_{\mathrm{K}}}\right)^{1 / 2} \tilde{J}_{\mathrm{i}} \equiv n_{\mathrm{K}^{+}} \nu_{\mathrm{K}^{+}, \mathrm{coll}}
$$

$\tilde{J}_{\mathrm{i}}$ being the focusing factor appropriate for ions (Draine \& Sutin 1987) and $m_{\mathrm{K}}$ the mass of a potassium atom, and

$$
\mathcal{R}_{\mathrm{K} 0, \mathrm{coll}}=n_{\mathrm{K}^{0}} n_{\mathrm{gr}} \pi a_{\mathrm{gr}}^{2}\left(\frac{8 k T}{\pi m_{\mathrm{K}}}\right)^{1 / 2} \equiv n_{\mathrm{K}^{0}} \nu_{\mathrm{K}^{0}, \mathrm{coll}} \cdot
$$

The ratio of bound $\mathrm{K}$ atoms to gas-phase $\mathrm{K}$ atoms can be found be balancing these two rates:

$$
\begin{gathered}
\frac{n_{\mathrm{K}, \mathrm{cond}}}{n_{\mathrm{K}}} \sim n_{\mathrm{gr}} \pi a_{\mathrm{gr}}^{2}\left(\frac{8 k T}{\pi m_{\mathrm{K}}}\right)^{1 / 2} S_{\mathrm{i}} \nu^{-1} \exp \left(+\frac{E_{\mathrm{a}}}{k T}\right) \\
=\rho_{\mathrm{g}} \frac{\left(\rho_{\mathrm{gr}} / \rho_{\mathrm{g}}\right)}{4 \rho_{\mathrm{s}} a_{\mathrm{gr}} / 3}\left(\frac{8 k T}{\pi m_{\mathrm{K}}}\right)^{1 / 2} S_{\mathrm{i}} \nu^{-1} \exp \left(+\frac{E_{\mathrm{a}}}{k T}\right) .
\end{gathered}
$$

The fraction of potassium atoms in the gas phase (versus bound in grains) is strongly temperature dependent.

At low temperatures, potassium is unlikely to be found in the gas phase because it will chemically react with silicates and be bound into minerals. In particular, potassium resides in feldspar $\left(\mathrm{KAlSi}_{3} \mathrm{O}_{8}\right)$ below its $50 \%$ condensation temperature $\approx 1006 \mathrm{~K}$ (Lodders 
2003). To mimic this behavior, we assume the binding energy of $\mathrm{K}$ atoms in silicates is $E_{\mathrm{a}}=3.26 \mathrm{eV}$, so that $n_{\mathrm{K}, \text { coll }} / n_{\mathrm{K}}=1 / 2$ at $T=1006 \mathrm{~K}$. Here we take a gas density $n_{\mathrm{H}_{2}}=10^{14} \mathrm{~cm}^{-3}$, a dust-to-gas ratio $=0.01$, a grain density $\rho_{\mathrm{s}}=3 \mathrm{~g} \mathrm{~cm}^{-3}$, a grain radius $a_{\mathrm{gr}}=1 \mu \mathrm{m}$, a sticking coefficient $S_{\mathrm{i}}=1$, and neglecting the charge state of the $\mathrm{K}$ atoms.

At temperatures above $1006 \mathrm{~K}$, potassium atoms will evaporate from the surface, but whether they leave as neutral atoms or as ions depends on their ionization potential and the work function of the grains, according to the Saha-Langmuir equation. Among departing potassium atoms, the ratio of ions to neutrals obeys

$$
\frac{n_{\mathrm{K}^{+}}}{n_{\mathrm{K}^{0}}}=\frac{g_{+}}{g_{0}} \exp \left(+\frac{W_{\mathrm{eff}}-\mathrm{IP}}{k T}\right) .
$$

The fraction of leaving $\mathrm{K}$ atoms that are positive ions is then

$$
f_{+}=\frac{\left(n_{\mathrm{K}^{+}} / n_{\mathrm{K}^{0}}\right)}{1+\left(n_{\mathrm{K}^{+}} / n_{\mathrm{K}^{0}}\right)} \text {. }
$$

The rate at which ions evaporate from grains (per volume of nebula) is therefore $\mathcal{R}_{\mathrm{K} \text {,evap }} f_{+}$, and the rate at which neutral $\mathrm{K}$ atoms do so is $\mathcal{R}_{\mathrm{K} \text {,evap }}\left(1-f_{+}\right)$.

\subsection{Chemical Network}

We now construct a chemical network to calculate the densities of the five species, $n_{\mathrm{e}}$ (free electrons), $n_{\mathrm{i}}$ (gas-phase atomic ions), $n_{\mathrm{K}, \text { coll }}$ (grain-adsorbed $\mathrm{K}$ atoms), $n_{\mathrm{K}^{+}}$ (gas-phase $\mathrm{K}$ ions), and $n_{\mathrm{K}^{0}}$ (gas-phase neutral $\mathrm{K}$ atoms), as well as the charge $Z$ on each 
dust grain. The time derivatives of these quantities are as follows.

$$
\begin{aligned}
\frac{d n_{\mathrm{i}}}{d t} & =+\zeta n_{\mathrm{H}_{2}}-\beta n_{\mathrm{e}} n_{\mathrm{i}}-\mathcal{R}_{\mathrm{i}, \text { ads }} \\
\frac{d n_{\mathrm{e}}}{d t} & =+\zeta n_{\mathrm{H}_{2}}-\beta n_{\mathrm{e}} n_{\mathrm{i}}-\mathcal{R}_{\mathrm{e}, \text { ads }}-\mathcal{R}_{\text {gas }, \text { rec }}+\mathcal{R}_{\text {gas,ion }}+\mathcal{R}_{\mathrm{therm}} \\
\frac{d n_{\mathrm{K}}}{d t} & =-\mathcal{R}_{\text {gas }, \text { rec }}+\mathcal{R}_{\text {gas,ion }}+\mathcal{R}_{\mathrm{K}, \text { evap }} f_{+}-\mathcal{R}_{\mathrm{K}+\text {, coll }} \\
\frac{d n_{\mathrm{K}}}{d t} & =+\mathcal{R}_{\text {gas,rec }}-\mathcal{R}_{\text {gas,ion }}+\mathcal{R}_{\mathrm{K}, \text { evap }}\left(1-f_{+}\right)-\mathcal{R}_{\mathrm{K} 0, \text { coll }} \\
\frac{d n_{\mathrm{K}, \text { cond }}}{d t} & =+\mathcal{R}_{\mathrm{K}+\text {,oll }}+\mathcal{R}_{\mathrm{K} 0, \text { coll }}-\mathcal{R}_{\mathrm{K}, \text { evap }}
\end{aligned}
$$

It is straightforward to confirm that the total number of potassium atoms (neutral, ionized, and bound) is conserved in such a model, i.e., $n_{\mathrm{K}, \text { cond }}+n_{\mathrm{K}^{+}}+n_{\mathrm{K}^{0}}=n_{\mathrm{K} \text {,tot }}$, where we assume $n_{\mathrm{K}, \text { tot }}=3.04 \times 10^{-7} n_{\mathrm{H}_{2}}$ (Lodders 2003). We seek a steady-state solution by setting all time derivatives to zero. Note that the five densities are, through the cross section modifications $\tilde{J}$, all functions of the grain charge $Z$, the sixth variable. The sixth equation needed to close the system corresponds to charge neutrality. If grains' mean charge is $+Z e$,

$$
n_{\mathrm{gr}} Z+n_{\mathrm{K}^{+}}+n_{\mathrm{Mg}^{+}}-n_{\mathrm{e}}=0 .
$$

In practice, we vary $Z$ till charge neutrality is achieved and the other densities are in steady state.

More specifically, we find the steady-state solution for gas of density $n_{\mathrm{H}_{2}}$ and temperature $T$ as follows. At a particular value of $Z$, we calculate the cross section modifications for ions, electrons, and neutral atoms, using the approximation formulas of Draine \& Sutin (1987). We assume the gas and dust temperatures are identical; in protoplanetary disks this is an excellent assumption except for the uppermost $\lesssim 0.1 \mathrm{~g} \mathrm{~cm}^{-2}$ surface layers (Glassgold et al. 2004). With the coefficients known, we then solve for the species' steady-state abundances. Given guesses for the densities of $\mathrm{K}^{0}$ and $\mathrm{K}^{+}$, we find the 
electron density by first solving for $n_{\mathrm{i}}$ :

$$
n_{\mathrm{i}}=\frac{\zeta n_{\mathrm{H}_{2}}}{\beta n_{\mathrm{e}}+n_{\mathrm{gr}} \nu_{, \mathrm{i}, \mathrm{ads}}}=\frac{\zeta n_{\mathrm{H}_{2}}}{\beta n_{\mathrm{e}}+n_{\mathrm{gr}} \pi a_{\mathrm{gr}}^{2} C_{\mathrm{i}} S_{\mathrm{i}} \tilde{J}_{\mathrm{i}}} .
$$

This value is then substituted into the equation for the electron density,

$$
\zeta n_{\mathrm{H}_{2}}+\mathcal{R}_{\mathrm{therm}}+k_{2} n_{\mathrm{H}_{2}} n_{\mathrm{K}^{0}}=n_{\mathrm{e}}\left[\beta n_{\mathrm{i}}+k_{-2} n_{\mathrm{H}_{2}} n_{\mathrm{K}^{+}}+\nu_{\mathrm{e}, \mathrm{ads}}\right]
$$

to derive a quadratic equation for $n_{\mathrm{e}}$ :

$$
\begin{gathered}
{\left[\beta\left(\nu_{\mathrm{e}, \mathrm{ads}}+k_{-2} n_{\mathrm{H}_{2}} n_{\mathrm{K}^{+}}\right)\right] n_{\mathrm{e}}^{2}} \\
+\left[\nu_{\mathrm{i}, \text { ads }}\left(\nu_{\mathrm{e}, \text { ads }}+k_{-2} n_{\mathrm{H}_{2}} n_{\mathrm{K}^{+}}\right)-\beta\left(n_{\mathrm{gr}} 4 \pi a_{\mathrm{gr}}^{2} j(T)+k_{2} n_{\mathrm{H}_{2}} n_{\mathrm{K}^{0}}\right)\right] n_{\mathrm{e}} \\
-\nu_{\mathrm{i}, \text { ads }}\left[\zeta n_{\mathrm{H}_{2}}+n_{\mathrm{gr}} 4 \pi a_{\mathrm{gr}}^{2} j(T)+k_{2} n_{\mathrm{H}_{2}} n_{\mathrm{K}^{0}}\right]=0 .
\end{gathered}
$$

Once $n_{\mathrm{e}}$ is found, $n_{\mathrm{i}}$ is found using the above equation, as are the other densities, as follows:

$n_{\mathrm{K}^{+}}=\frac{n_{\mathrm{K}, \text { tot }}\left(k_{2} n_{\mathrm{H}_{2}}+\nu_{\mathrm{K}^{0}, \text { coll }} f^{+}\right)}{k_{2} n_{\mathrm{H}_{2}}\left(1+\frac{\nu_{\mathrm{K}}+\text { coll }}{\nu_{\text {evap }}}\right)+k_{-2} n_{\mathrm{H}_{2}} n_{\mathrm{e}}\left(1+\frac{\nu_{\mathrm{K}^{0}, \text { coll }}}{\nu_{\text {evap }}}\right)+\nu_{\mathrm{K}^{+}, \text {coll }}\left(1-f^{+}\right)+\nu_{\mathrm{K}^{0}, \text { coll }} f^{+}+\frac{\nu_{\mathrm{K}^{0}, \text { coll }} \nu_{\mathrm{K}^{+}, \text {coll }}}{\nu_{\text {evap }}}}$

yields the $\mathrm{K}^{+}$density, in terms of which

$$
n_{\mathrm{K}^{0}}=\frac{n_{\mathrm{K}, \mathrm{tot}}-n_{\mathrm{K}^{+}}\left(1+\frac{\nu_{\mathrm{K}}+, \mathrm{coll}}{\nu_{\mathrm{evap}}}\right)}{1+\frac{\nu_{\mathrm{K}^{0}, \mathrm{coll}}}{\nu_{\mathrm{evap}}}}
$$

and

$$
n_{\mathrm{K}, \mathrm{cond}}=\frac{n_{\mathrm{K}, \mathrm{tot}}-n_{\mathrm{K}^{+}}\left(1-\frac{\nu_{\mathrm{K}^{+}, \mathrm{coll}}}{\nu_{\mathrm{K}^{0}, \mathrm{coll}}}\right)}{1+\frac{\nu_{\mathrm{evap}}}{\nu_{\mathrm{K}^{0}, \mathrm{coll}}}} .
$$

In practice, at each guess for $Z$ we iterate between solving for $n_{\mathrm{e}}$ and solving for $n_{\mathrm{i}}, n_{\mathrm{K}^{0}}$ and $n_{\mathrm{K}^{+}}$, then updating $n_{\mathrm{e}}$. Performing 10 such iterations allows us to compute the net charge $n_{\mathrm{i}}+n_{\mathrm{K}^{+}}+n_{\mathrm{gr}} Z-n_{\mathrm{e}}$ for the given $Z$. At very large $Z>0$ the net charge is positive, while at very large $Z<0$ it is negative, so it goes to zero at some finite $Z$. We use a bisection method with 80 steps to find the value of $Z$ that yields both charge neutrality and ionization equilibrium. 


\section{Results}

\subsection{Canonical Case}

We have solved for the abundances of electrons, ions $\left(\mathrm{Mg}^{+}\right.$and $\left.\mathrm{K}^{+}\right)$, as well as neutral and bound $\mathrm{K}$ atoms, using the chemical network described above. We start with our canonical case, characterized by $n_{\mathrm{H}_{2}}=10^{14} \mathrm{~cm}^{-3}$, grains with a dust-to-gas mass ratio 0.01 , uniform radius $a_{\mathrm{gr}}=1 \mu \mathrm{m}$, and work function $W=5.0 \mathrm{eV}$. Figure 2 shows the fractional abundances of electrons, $x_{\mathrm{e}}=n_{\mathrm{e}} / n_{\mathrm{H}_{2}}$, as well as $x_{\mathrm{i}}, x_{\mathrm{K}^{+}}, x_{\mathrm{K}^{0}}$, and $x_{\mathrm{K} \text {,cond }}$, as functions of temperature. In cold gas, $\lesssim 500 \mathrm{~K}$, the ion and electron densities come from the balance between ionizations by energetic particles and collisions with dust grains. Because of their slower thermal velocities, ions take longer to reach the grains and are more abundant, with $x_{\mathrm{i}} \sim 10^{-19}$, compared to $x_{\mathrm{e}} \sim 10^{-21}$ for electrons. The very low abundances are a consequence of the low ionization rates associated with the radioactivities. Essentially all potassium is bound into solids, with few ions or neutral atoms in the gas phase. As the temperature increases, however, $\mathrm{K}^{0}$ and especially $\mathrm{K}^{+}$enter the gas phase. Some come directly from evaporation of $\mathrm{K}^{+}$ions from grain surfaces; because $\mathrm{IP}<W$, most $\mathrm{K}$ atoms leave as ions. In addition, once the temperatures exceed about $750 \mathrm{~K}$, electrons are produced effectively by thermionic emission. Above $1000 \mathrm{~K}$, the potassium atoms evaporate from solids; most takes the form of neutral $\mathrm{K}^{0}$ atoms in the gas-phase. Only in gas hotter than about $1500 \mathrm{~K}$ do the $\mathrm{K}^{+}$ions compare in abundance to the neutral $\mathrm{K}^{0}$ atoms. 


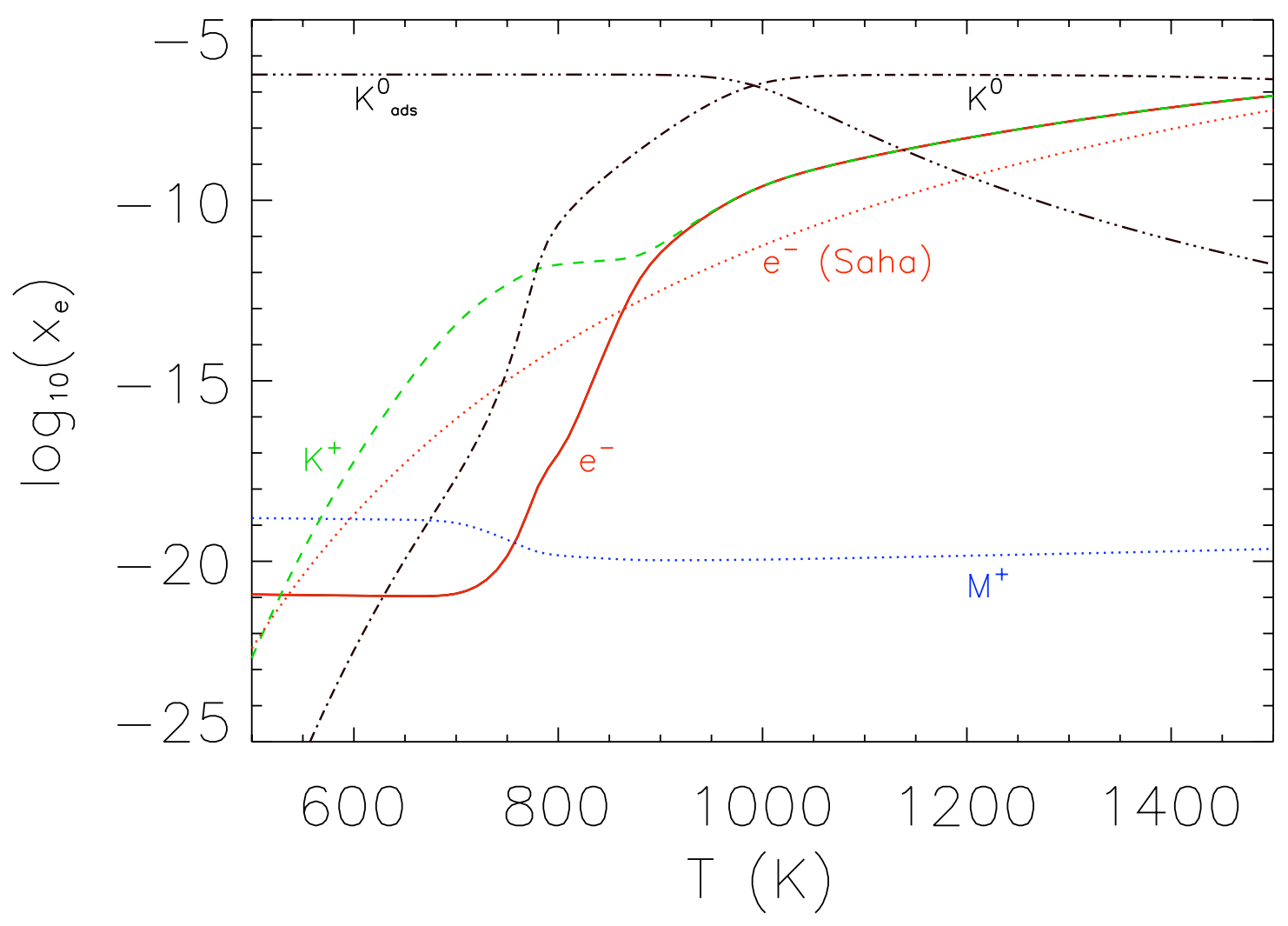

Fig. 2.- Abundances of electrons (solid red curve), atomic (Mg) ions (dotted blue curve), $\mathrm{K}^{+}$ions (dashed green curve), gas-phase $\mathrm{K}^{0}$ atoms (dash-dot curve), and bound $\mathrm{K}$ atoms (dash-dot-dot-dot curve), as functions of temperature $T$, for the canonical case (gas density $n_{\mathrm{H}_{2}}=10^{14} \mathrm{~cm}^{-3}$, dust-to-gas mass ratio 0.01 , grain work function $W=5.0 \mathrm{eV}$. At low temperatures, non-thermal ionizations (from radioisotopes) dominate, but emission of $\mathrm{K}^{+}$ ions from dust grains is significant for $T \gtrsim 550 \mathrm{~K}$. Evaporation of $\mathrm{K}^{0}$ atoms from grains, and their subsequent thermal ionization, contribute significantly to $\mathrm{K}^{+}$and electron densities for $T \gtrsim 750 \mathrm{~K}$. For $T \gtrsim 1000 \mathrm{~K}$, thermal ionizations and recombinations dominate and are nearly in thermodynamic equilibrium. For comparison, we also plot the electron abundance derived using the Saha equation (dashed red curve). The Saha equation overpredicts the ionization at temperatures $\lesssim 800 \mathrm{~K}$, and underpredicts the ionization at higher temperatures. 
The bolded curve in Figure 3 shows the charge on dust grains as a function of temperature. Below about $700 \mathrm{~K}$, when the ionization rate is low, grains typically hold only a few electron charges. The value of $Z$ is such that charging by electron and ion collisions balance each other, including the effects of charge on the collision rates (i.e., $\tilde{J}_{\mathrm{e}}$, $\left.\tilde{J}_{\mathrm{i}}\right)$. As the temperature increases, however, ion emission comes to dominate the charging, and the grains quickly become negatively charged; at $900 \mathrm{~K}$ the charge is about $-700 e$. Because adsorption of gas-phase ions is unimportant, the grains charge until $W_{\text {eff }}$ is reduced enough that ion emission is suppressed and thermionic emission enhanced. In other words, ions are emitted until $\left|Z e^{2} / a_{\mathrm{gr}} W\right|$ approaches unity, which requires $Z \sim 3500$. At $900 \mathrm{~K}$, $\left|Z e^{2} / a_{\mathrm{gr}}\right| \approx 0.2 \mathrm{~W}$. As the temperature increases, collisions of ions with grain surfaces begin to matter, and the grain charge is reduced, approaching the thermodynamic limit where $\left|Z e^{2} / a_{\mathrm{gr}}\right| \sim k T$, or $Z \sim-100($ at $1500 \mathrm{~K})$. 


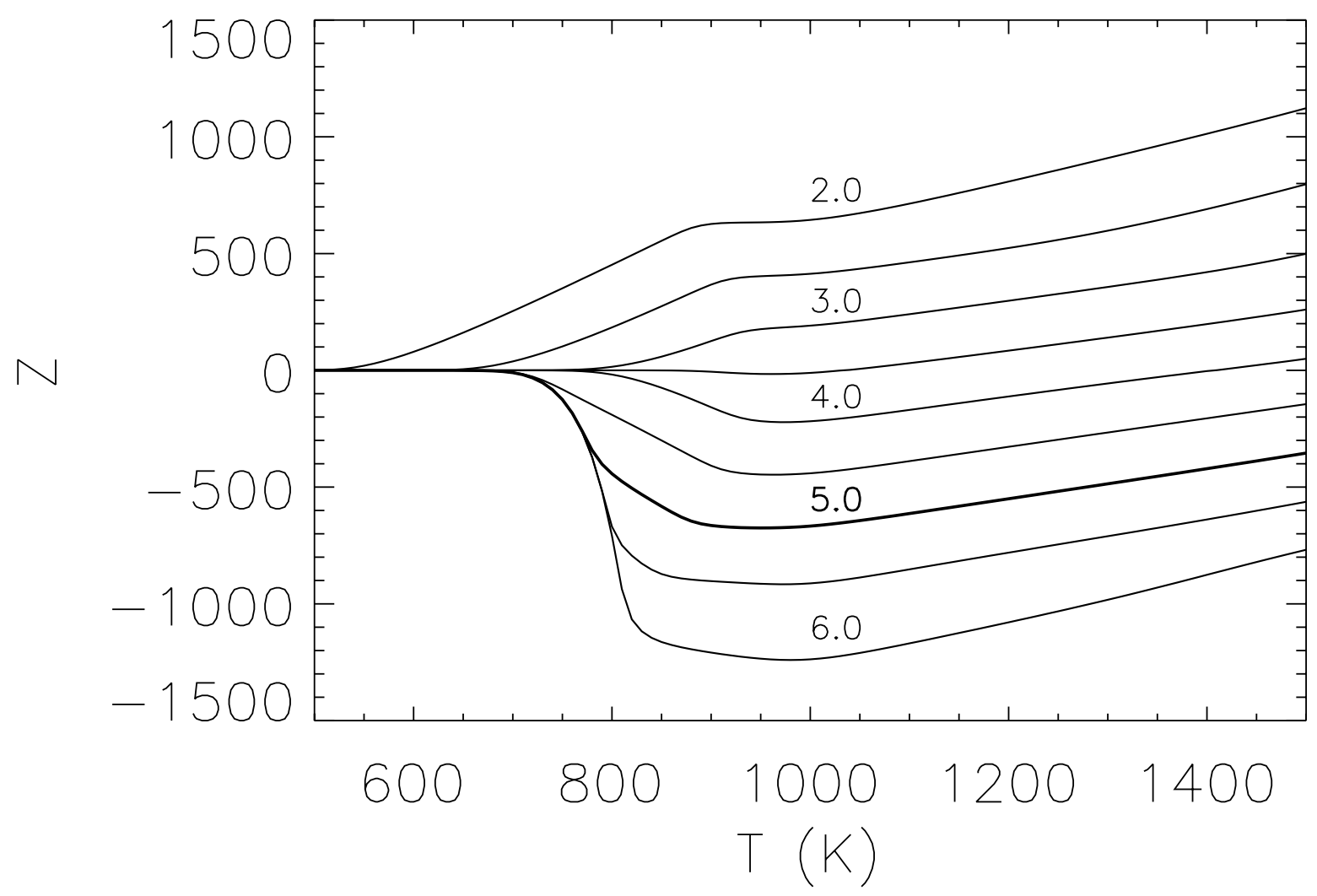

Fig. 3.- Charge on dust grains (in units of elementary charge $e$ ) as a function of temperature, for the canonical case but with various grain work functions. From top to bottom, $W=2.0,2.5, \ldots 6.0 \mathrm{eV}$. The bolded curve corresponds to $W=5.0 \mathrm{eV}$. For low work functions ( $W \lesssim 4 \mathrm{eV}$, thermionic emission dominates the charging, and dust grains are positively charged; as temperature increases, thermionic emission becomes more effective, and grains become more positively charged. For large work functions ( $W \gtrsim 4 \mathrm{eV}$ ), ion emission dominates, and grains become negatively charged; as the temperature increases, collisions of $\mathrm{K}^{+}$ions onto the grains becomes more effective, and grains become less negatively charged.

The relative abundances of neutral and ionized $\mathrm{K}$ atoms also approach the 
thermodynamic limit only at high temperatures. The bolded curve in Figure 4 shows what effective ionization potential energy $E$ must exist so that the Saha equation would yield the correct ratio for $n_{\mathrm{K}}+n_{\mathrm{e}} / n_{\mathrm{K}^{0}}$, i.e.,

$$
E \equiv-\left[\ln \left(n_{\mathrm{K}^{+}} n_{\mathrm{e}} / n_{\mathrm{K}^{0}}\right)-35.42\right] k T .
$$

At high temperatures it tends to approach the actual value of IP for potassium, $4.34 \mathrm{eV}$, but at lower temperatures $E$ is lower, reflecting the fact that many $\mathrm{K}$ atoms evaporate as $\mathrm{K}^{+}$ ions from grain surfaces, according to the Saha-Langmuir equation. At all temperatures, but especially below $800 \mathrm{~K}$, emissions from dust grains generate more $\mathrm{K}^{+}$ions than the Saha equation would indicate. 


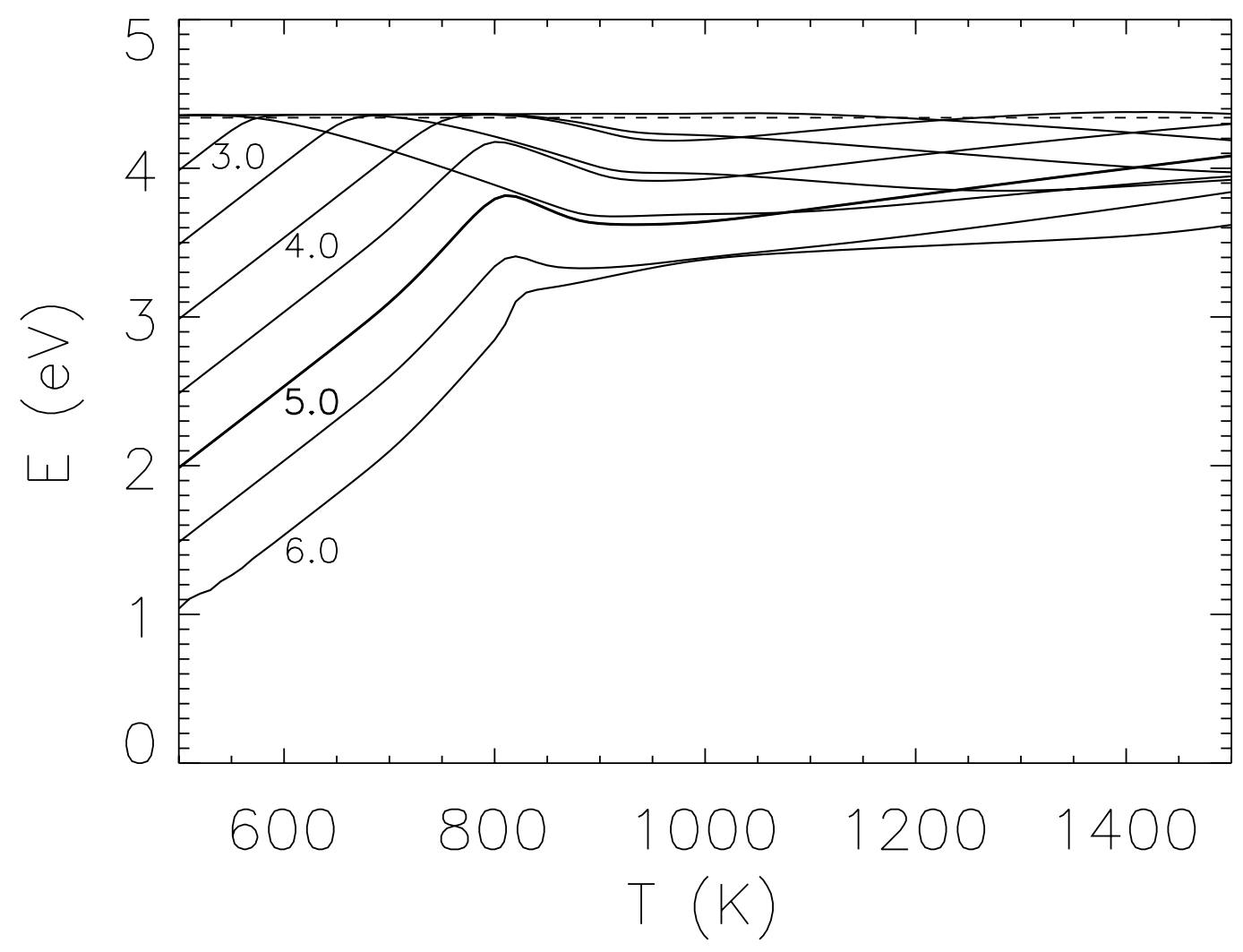

Fig. 4.- Effective ionization potential, E, needed for the Saha equation to return the correct value of $n_{\mathrm{K}}+n_{\mathrm{e}} / n_{\mathrm{K}^{0}}$, as a function of temperature, for various values of the work function, $W$. The value of $W$ (in eV) is used to label several of the curves. The case $W=5.0 \mathrm{eV}$ is bolded. The horizontal dashed line refers to $E=4.34 \mathrm{eV}$, the value tht would obtain in the absence of species other than potassium. Because of emission of charges from dust grains (thermionic emission of electrons at low $W$, ion emission of $\mathrm{K}^{+}$at large $W$ ), the gas charge state is always greater than expected based on a pure Saha equation for potassium; that is, $E<4.34 \mathrm{eV}$. The effect is most pronounced and sensitive to $W$ at low temperatures $\lesssim 800 \mathrm{~K}$. For $T \gtrsim 800 \mathrm{~K}, 3.5 \mathrm{eV} \lesssim E \lesssim 4.34 \mathrm{eV}$ for $W$ between 2 and $6 \mathrm{eV}$. For work functions close to the ionization potential of potassium $(W \approx 4.34 \mathrm{eV}), E \approx 4.34 \mathrm{eV}$. 
Figure 5 shows the timescales for the steady-state electron density $n_{\mathrm{e}}$ to be affected by various ionization / recombination processes, as functions of temperature. For example, since $\mathcal{R}_{\text {therm }}$ is the rate per volume of nebula at which electrons are produced, $t_{\text {therm }}=n_{\mathrm{e}} / \mathcal{R}_{\text {therm }}$. Likewise, the timescale for recombination of electrons with atomic ions is defined as $n_{\mathrm{e}} /\left(\beta n_{\mathrm{e}} n_{\mathrm{i}}\right)=1 /\left(\beta n_{\mathrm{i}}\right)$. At low temperatures, $T<700 \mathrm{~K}$, the dominant loss mechanism is adsorption of electrons onto dust grains, while the dominant production mechanism is ionizations of gas-phase molecules by energetic particles. These balance each other and the electron density turns over on timescales of roughly 10 seconds. At temperatures above $800 \mathrm{~K}$, thermionic emission of electrons from grains dominates, and the electron density increases. Ionizations by energetic particles become less important compared to thermionic emission. But because the electron density is greater, the timescale for overturning the electron population rises, to above 1 hour at about $780 \mathrm{~K}$, to about 1 day at $870 \mathrm{~K}$, and peaking at $10^{6} \mathrm{~s}$ at $900 \mathrm{~K}$. At higher temperatures still, the timescales for both adsorption and thermionic emission decrease, eventually falling below about 1 hour again for $T \gtrsim 1300 \mathrm{~K}$. 


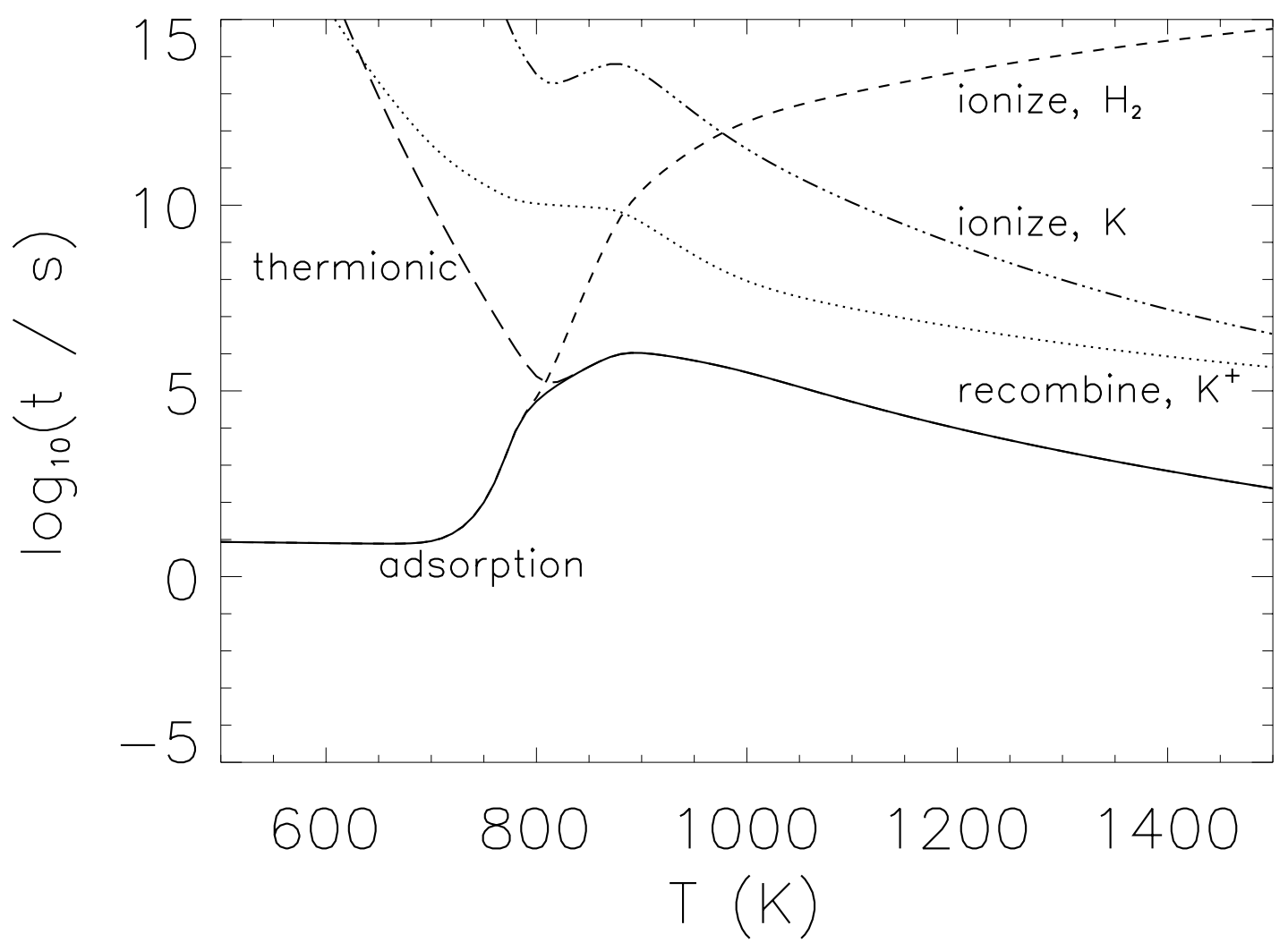

Fig. 5.- Characterisic timescales for various processes affecting the electron abundances, as functions of temperature, for our canonical case. The dominant removal processes is always electrons colliding with grains ('adsorption', solid curve). At low temperatures ( $T \lesssim 800 \mathrm{~K}$ ), ionization of $\mathrm{H}_{2}$ by energetic particles ('ionize, $\mathrm{H}_{2}$ ', dashed curve) is the dominant charging process. At higher temperatures, emission of electrons from grains ('thermionic', long dashed curve) dominates. At high temperatures the timescales for thermal ionization of K (dasheddotted curve) and recombinations of $\mathrm{K}^{+}$ions and electrons (dotted curve) become shorter but never dominate. Recombination of atomic ions and electrons is slower than all other processes and is not plotted. Notably, for temperatures $800 \mathrm{~K} \lesssim T \lesssim 1300 \mathrm{~K}$, the chemical timescales are longer than hours. 


\subsection{Variation with Work Function}

We now investigate the effect of varying the work function of the solids making up the grains, keeping other parameters the same as our canonical case $\left(n_{\mathrm{H}_{2}}=10^{14} \mathrm{~cm}^{-3}\right.$, dust-to-gas ratio $10^{-2}$ ). Figure 3 shows the charge on each dust grain (in units of $e$ ) as a function of temperature $T$ for various $W$. At low work function, $W \lesssim 4 \mathrm{eV}$, thermionic emission of electrons is very effective, whereas any potassium atoms leaving are likely to become neutral $\mathrm{K}^{0}$ atoms. The grains are positively charged. At higher temperatures, the rate of thermionic emission is greater, and the dust grains are increasingly positively charged. For high work function, $W \gtrsim 4 \mathrm{eV}$, thermionic emission is ineffective, but emission of $\mathrm{K}^{+}$is effective. At higher temperatures, grains emit ions more effectively and so are more negatively charged. At temperatures $\approx 800$ to $1000 \mathrm{~K}$, however, ion emission is as effective as it can be. At still higher temperatures, gas-phase ions' collisions with dust grains are increasingly important, and the grains become less negatively charged.

Figure 4 shows the effective ionization potential, $E$, needed for the Saha equation to yield our calculated value of $n_{\mathrm{K}}+n_{\mathrm{e}} / n_{\mathrm{K}^{0}}$, as a function of temperature, for various work functions $W$. At low temperatures this ratio depends sensitively on $W$, as the ion and electron abundances are controlled by grain processes. Especially for high work functions, $W \gtrsim 4 \mathrm{eV}$, ion emission dominates and increases $n_{\mathrm{K}^{+}}$above the levels predicted using the Saha equation. At low work functions, thermionic emission increases $n_{\mathrm{e}}$. In gas hotter than about $800 \mathrm{~K}$, thermal ionizations dominate and $E$ is driven toward the ionization potential $\mathrm{IP}=4.34 \mathrm{eV}$; however, this is only a trend, and the effective ionization potential falls in the range $3.5 \mathrm{eV} \lesssim E \lesssim 4.34 \mathrm{eV}$. The departure of $E$ from IP is most pronounced for $W$ that are far from IP; for $W \approx 4.34 \mathrm{eV}, E \approx 4.34 \mathrm{eV}$. Notably, though, $E$ never appreciably exceeds IP, meaning the dust makes the gas better-ionized than would be predicted using the Saha equation alone. 
Figure 6 shows the abundances of various species if the work function is increased to $W=6.0 \mathrm{eV}$. Compared to the case with $W=5.0 \mathrm{eV}$, the abundances as a function of temperature $T$ are quite similar, with the notable differences that $n_{\mathrm{K}^{0}}$ is much reduced, and $n_{\mathrm{K}^{+}}$rises with $T$ much more steeply. This underscores that what is driving up $n_{\mathrm{K}^{+}}$ for $T \lesssim 800 \mathrm{~K}$ is ions evaporating from grains, and whether the $\mathrm{K}$ atoms leave as ions or neutral atoms is a strong function of $W$, or more precisely $W-$ IP. Because of the grains' large negative charge, atomic ions' abundances are suppressed. 


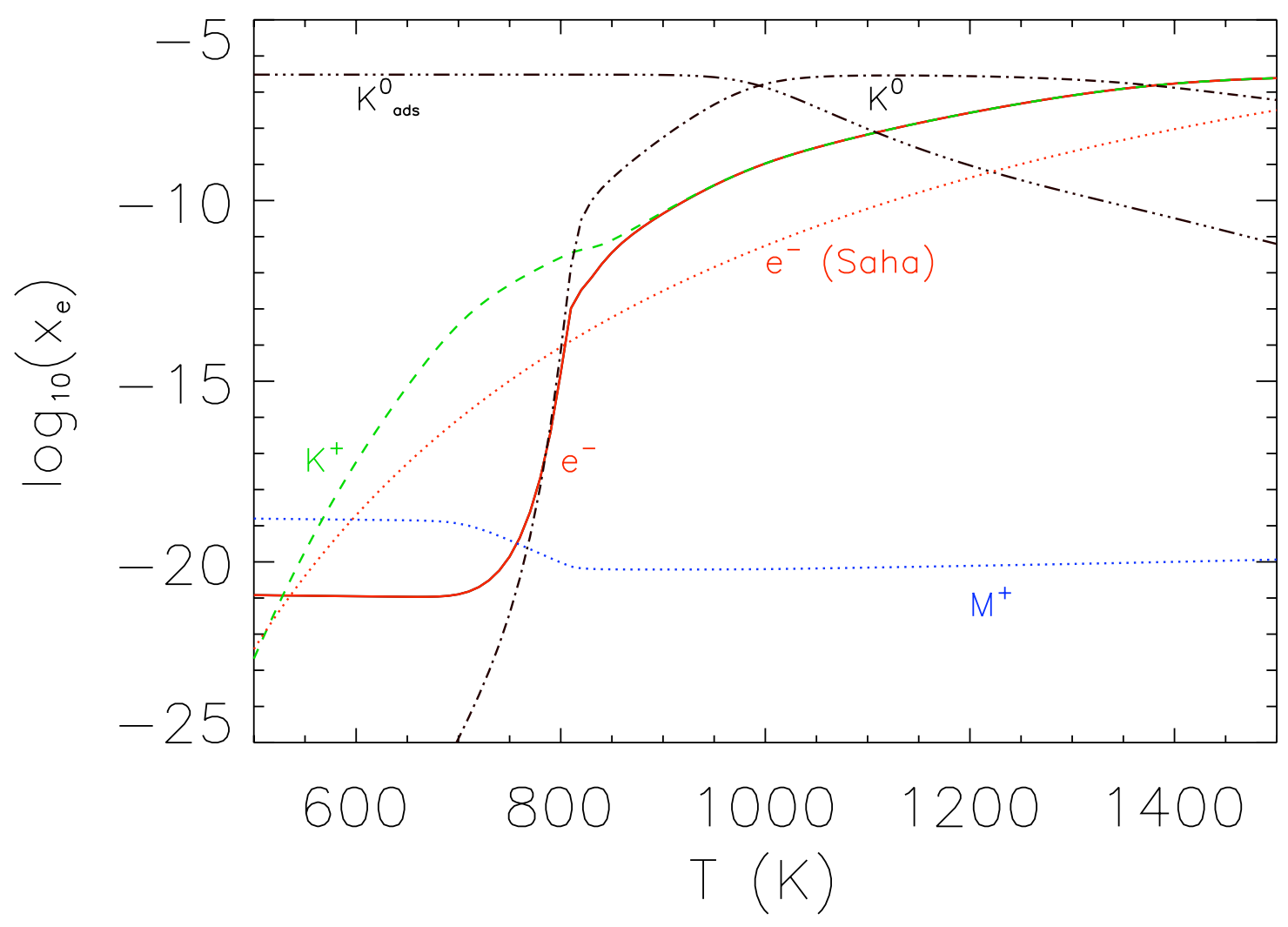

Fig. 6.- Abundances of electrons (solid red curve), atomic ( $\mathrm{Mg}$ ) ions (dotted blue curve), $\mathrm{K}^{+}$ ions (dashed green curve), gas-phase $\mathrm{K}^{0}$ atoms (dash-dot curve), and bound $\mathrm{K}$ atoms (dashdot-dot-dot curve), as functions of temperature $T$, for the case with canonical parameters (see Figure 2), but with grain work function $W=6.0 \mathrm{eV}$. The electron fraction predicted using the Saha equation is shown for comparison (dashed red curve).

Figure 7 shows the abundances if the work function is decreased to $W=2.0 \mathrm{eV}$. Again, the general behavior with temperature is similar, except for $T \lesssim 800 \mathrm{~K}$. For the $W=2.0 \mathrm{eV}$ case, production of $\mathrm{K}^{+}$ions is considerably lower; according to the Saha-Langmuir equation, nearly all $\mathrm{K}$ atoms leave as neutral atoms. On the other hand, production of free electrons 
by thermionic emission is much more effective. The electron fraction rises to $x_{\mathrm{e}} \sim 10^{-12}$ and plateaus there for temperatures between about $550 \mathrm{~K}$ and $900 \mathrm{~K}$. Because of the large positive charge on each grain, the gas-phase ions' abundances are elevated.

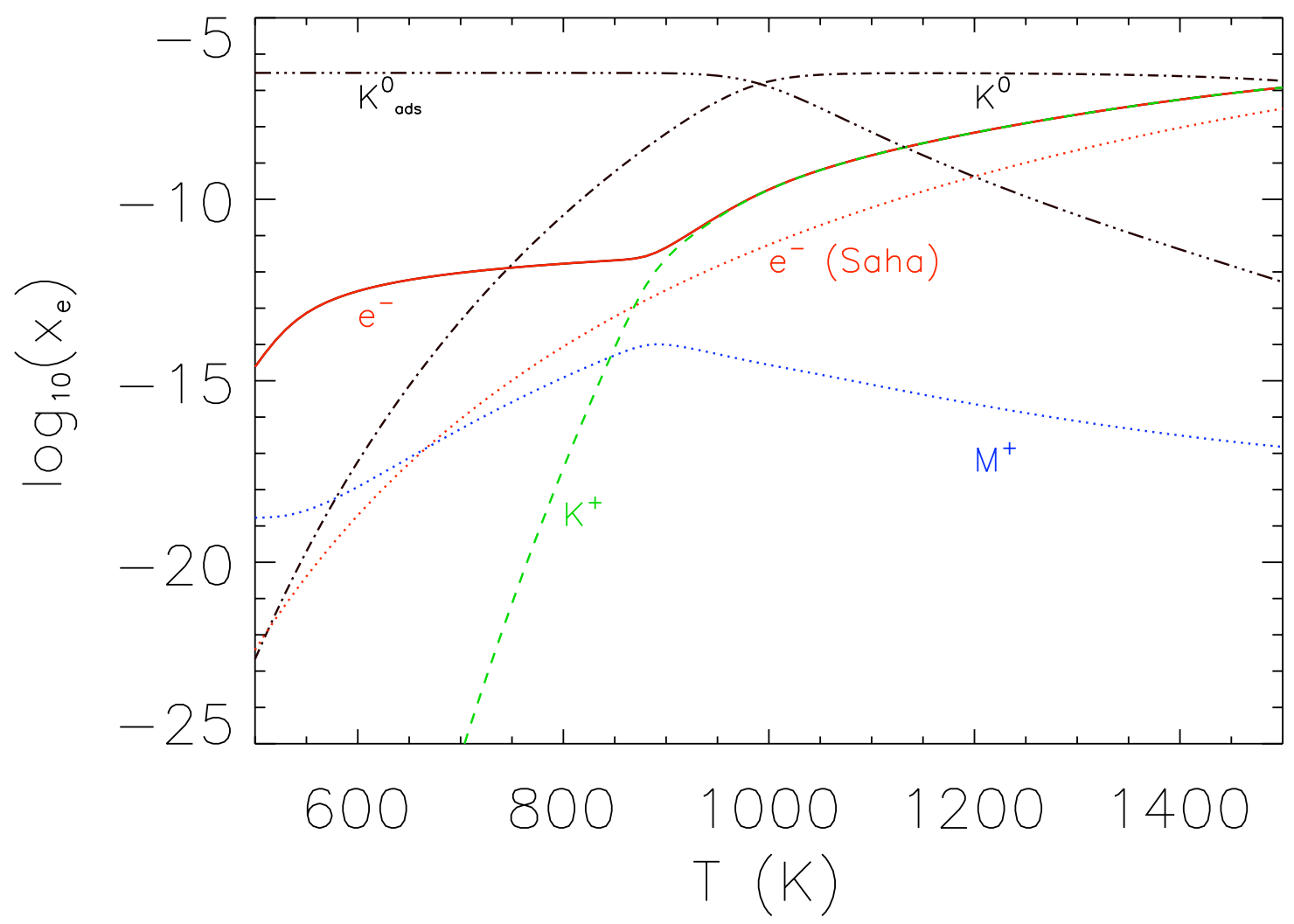

Fig. 7.- Abundances of electrons (solid red curve), atomic (Mg) ions (dotted blue curve), $\mathrm{K}^{+}$ ions (dashed green curve), gas-phase $\mathrm{K}^{0}$ atoms (dash-dot curve), and bound $\mathrm{K}$ atoms (dashdot-dot-dot curve), as functions of temperature $T$, for the case with canonical parameters (see Figure 2), but with grain work function $W=2.0 \mathrm{eV}$. The electron fraction predicted using the Saha equation is shown for comparison (dotted red curve).

Finally, Figure 8 shows just the electron abundance as a function of temperature, for 
various values of the grain work function. At high temperatures, $\gtrsim 1000 \mathrm{~K}$, the behavior of $x_{\mathrm{e}}$ with $T$ is very similar, but the exact value does depend on the work function, although not monotonically with $W$. At lower temperatures the electron fraction is especially sensitive to the work function, being up to 8 orders of magnitude higher for low values $(W \approx 2 \mathrm{eV})$ than for high values $(W \approx 6 \mathrm{eV})$. 


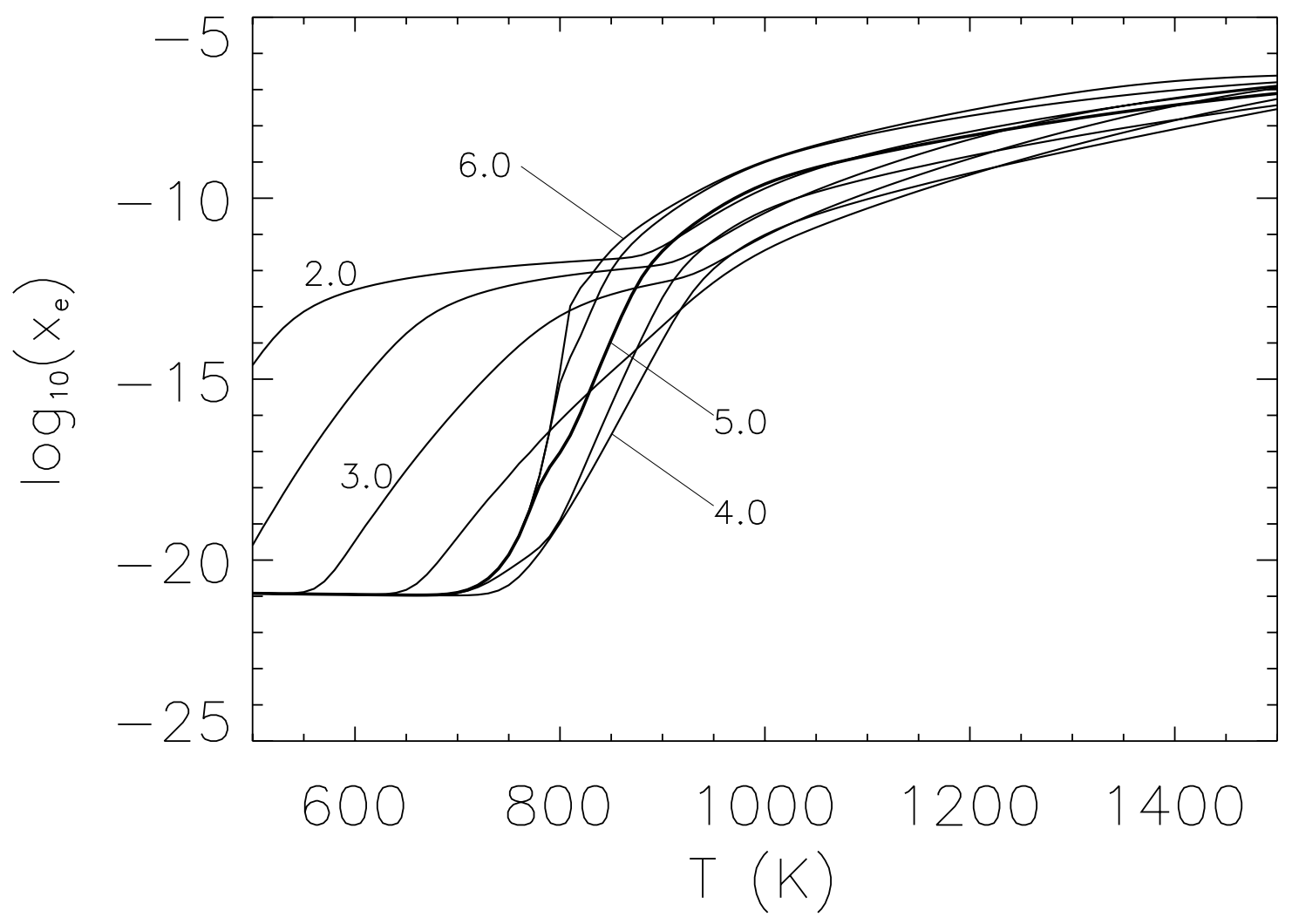

Fig. 8.- Electron fraction as a function of temperature, for various grain work functions. Other parameters are held at their values in the canonical case. The fiducial case ( $W=$ $5.0 \mathrm{eV})$ is in bold. Electron fractions at low temperature $(\lesssim 800 \mathrm{~K})$ decrease as the work function is increased from $2.0 \mathrm{eV}$ to $3.0 \mathrm{eV}$ to $4.0 \mathrm{eV}$ (as labeled). As the work function is increased more, from 4 to $6 \mathrm{eV}$, the electron fraction tends to increase for temperatures $T \approx 800-900 \mathrm{~K}$. 


\subsection{Variation with Dust-to-Gas Ratio}

We now investigate the effect of varying the dust-to-gas ratio, keeping other parameters the same as our canonical case $\left(W=5 \mathrm{eV}, n_{\mathrm{H}_{2}}=10^{14} \mathrm{~cm}^{-3}\right)$. Figure 9 shows the abundances of various species for the case of a dust-to-gas ratio 0.0001, a factor 100 below the canonical value. Figure 10 shows the abundances of various species for a dust-to-gas ratio of unity, a factor 100 higher than the canonical value. Comparing Figures 9 and 2, for the reduced dust-to-gas ratio both the electron and ion densities are higher at low temperatures $(T<600 \mathrm{~K})$, because both are produced by energetic particles and lost from the gas phase by adsorption onto grains. Comparing Figures 10 and 2, for the increased dust-to-gas ratio these densities are reduced below the canonical case. At high temperatures $(T \gtrsim 1000 \mathrm{~K})$, all three dust-to-gas ratios lead to similar electron densities, and $n_{\mathrm{e}} \approx n_{\mathrm{K}^{+}}$, because in that regime thermal ionizations of potassium dominate. At intermediate temperatures, there is a strong tendency for the electron and ion (both atomic ions and $\mathrm{K}^{+}$) densities to decrease with increasing dust-to-gas ratio, which makes sense given that they are lost mostly to collisions with dust grains. 


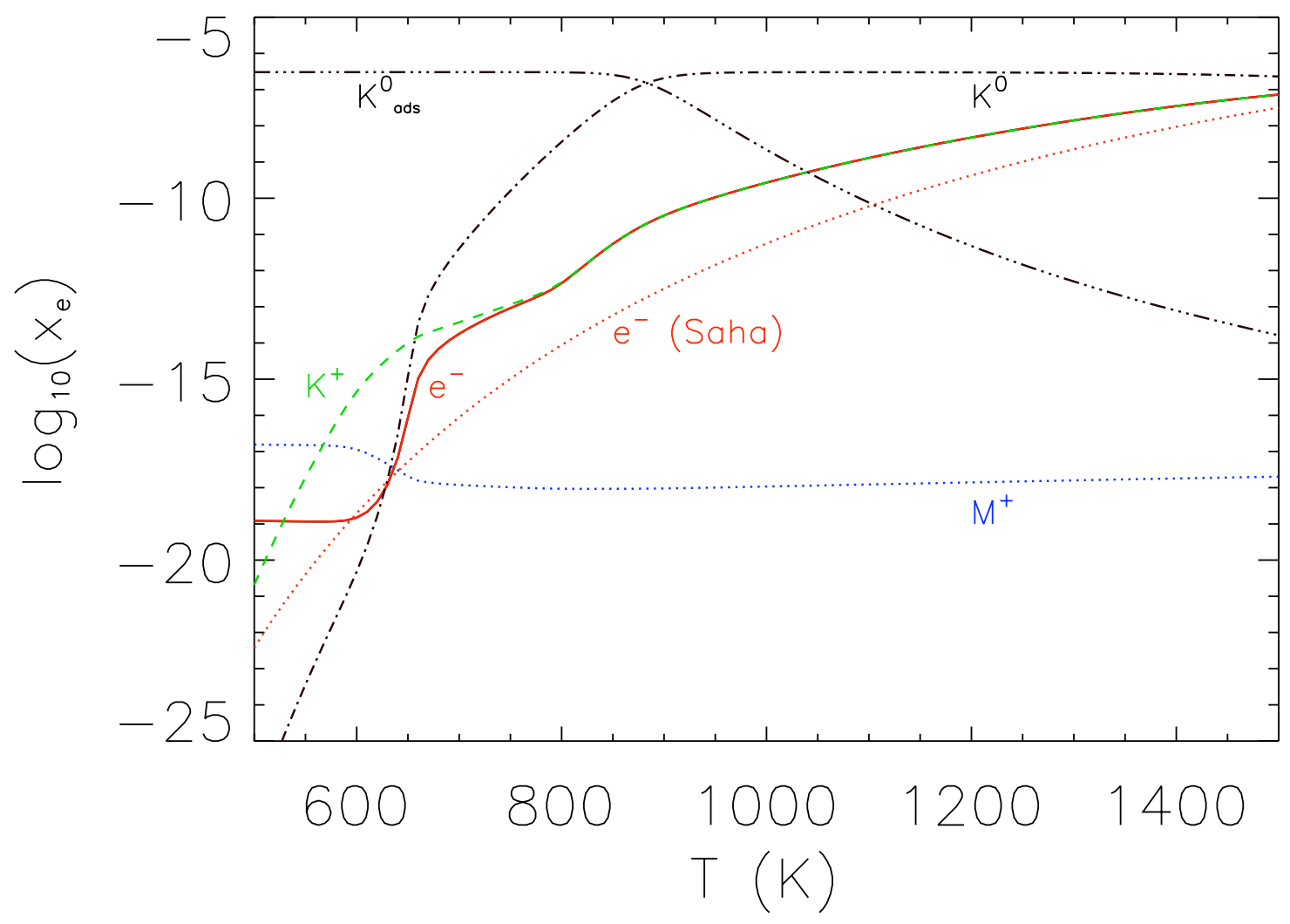

Fig. 9.- Abundances of electrons (solid curve), atomic ( $\mathrm{Mg}$ ) ions (dotted curve), $\mathrm{K}^{+}$ions (dashed curve), gas-phase $\mathrm{K}^{0}$ atoms (dash-dot curve), and bound $\mathrm{K}$ atoms (dash-dot-dot-dot curve), as functions of temperature $T$, for the case with canonical parameters (see Figure 2 ), but with dust-to-gas mass ratio $10^{-4}$. The electron fraction predicted using the Saha equation (dotted red curve) is shown for comparison. 


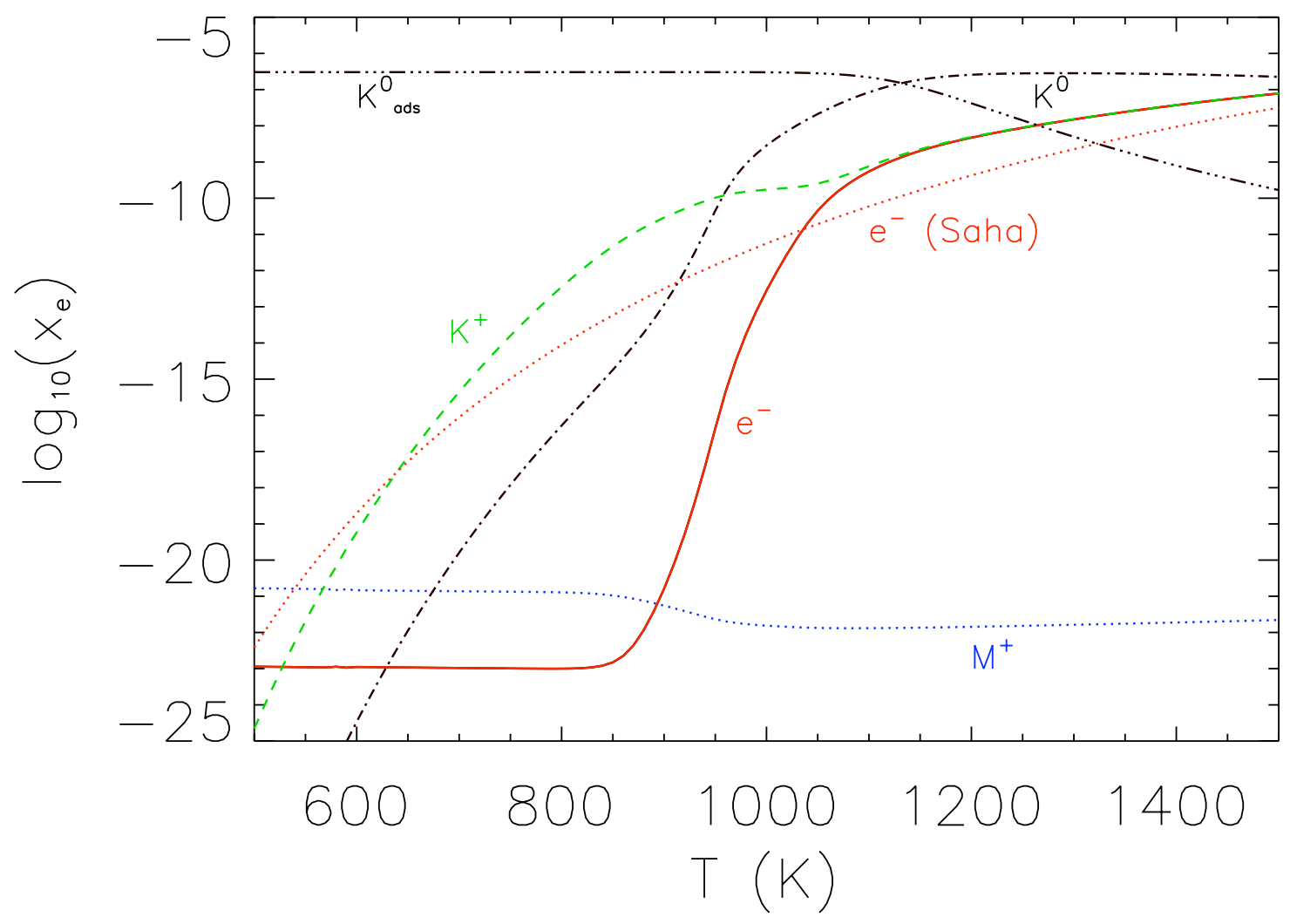

Fig. 10.- Abundances of electrons (solid curve), atomic (Mg) ions (dotted curve), $\mathrm{K}^{+}$ions (dashed curve), gas-phase $\mathrm{K}^{0}$ atoms (dash-dot curve), and bound $\mathrm{K}$ atoms (dash-dot-dot-dot curve), as functions of temperature $T$, for the case with canonical parameters (see Figure 2), but with dust-to-gas mass ratio 1. The electron fraction predicted using the Saha equation (dotted red curve) is shown for comparison.

Figure 11 shows the grain charge as a function of temperature, for various dust-to-gas ratios between $10^{-4}$ and unity. For all these cases, the charge on each grain is small at low temperatures, but becomes sharply negative as the temperature increases above some threshold; for the canonical ratio $10^{-2}$ that threshold temperature is $700 \mathrm{~K}$. As 
the temperature is increased further, the grain attains a maximum negative charge but then becomes less charged, eventually (above $1000 \mathrm{~K}$ in the canonical case) following $Z \approx-500(T / 1200 \mathrm{~K})^{-1}$ in all cases. For other dust-to-gas ratios the behaviors are the same but the thresholds occur at temperatures $100 \mathrm{~K}$ lower if the dust-to-gas ratio is $10^{-4}$, or $200 \mathrm{~K}$ higher if the dust-to-gas ratio is unity.

Finally, Figure 12 shows the electron fraction as a function of temperature for various values of the dust-to-gas ratio. As might be expected, $x_{\mathrm{e}}$ is inversely proportional to this ratio at low temperatures $(\lesssim 600 \mathrm{~K})$, and independent of this ratio at high temperatures ( $\gtrsim 1100 \mathrm{~K}$ ) where gas-phase reactions dominate both ionization and recombination. In between, the electron fraction is highly sensitive to the dust-to-gas ratio, being as much as 12 orders of magnitude higher for small values $\left(10^{-4}\right)$, as compared to large values (unity). 


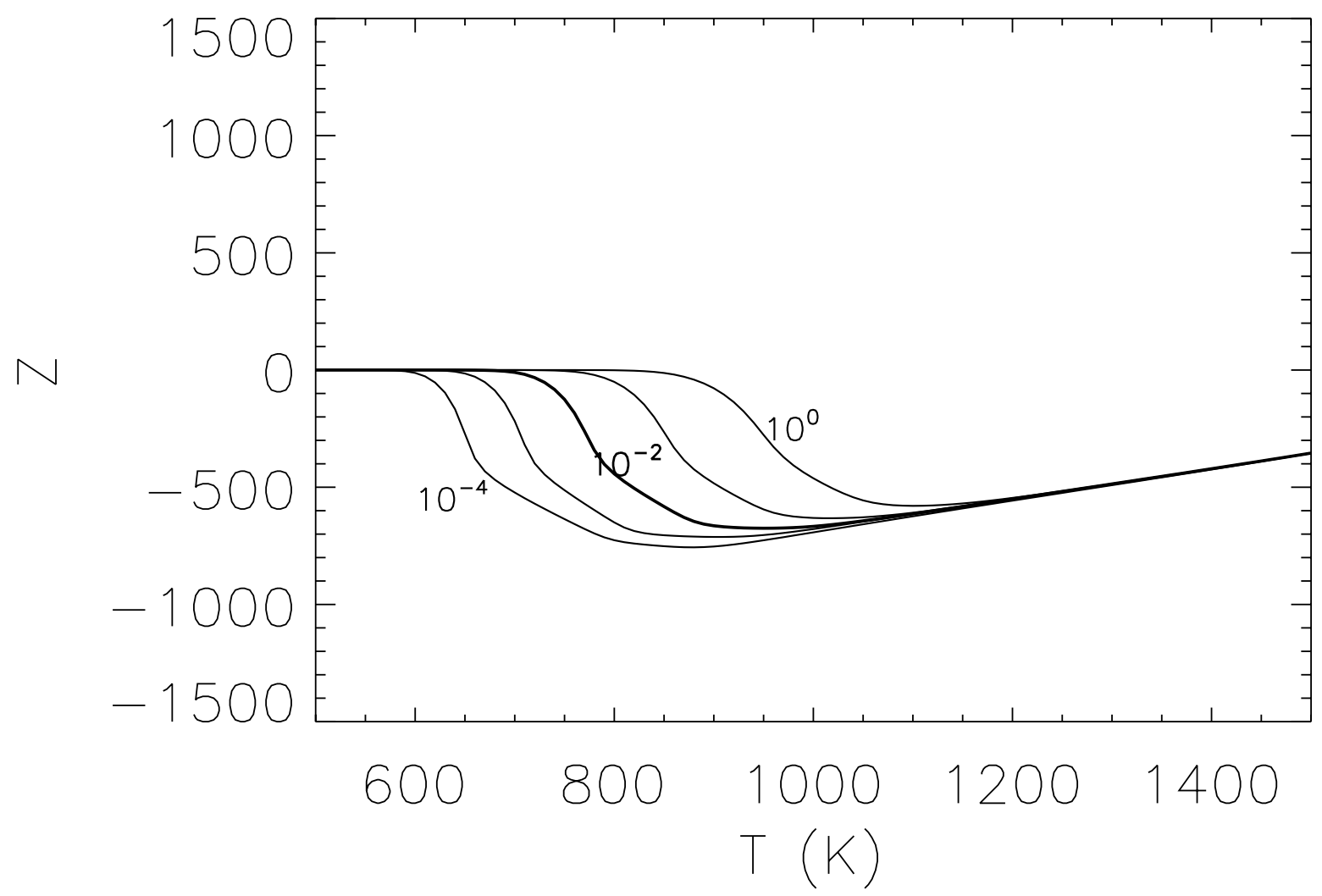

Fig. 11. - Grain charge (in units of elementary charge e), as a function of temperature $T$, for various dust-to-gas ratios, from left to right $10^{-4}, 10^{-3}, 10^{-2}, 10^{-1}$, and $10^{0}$. The curve for the canonical dust-to-gas ratio, $10^{-2}$, is bolded. With increasing temperature, the grains first become more negatively charged as they emit $\mathrm{K}^{+}$ions, then become less negatively charged as they collide with ions. 


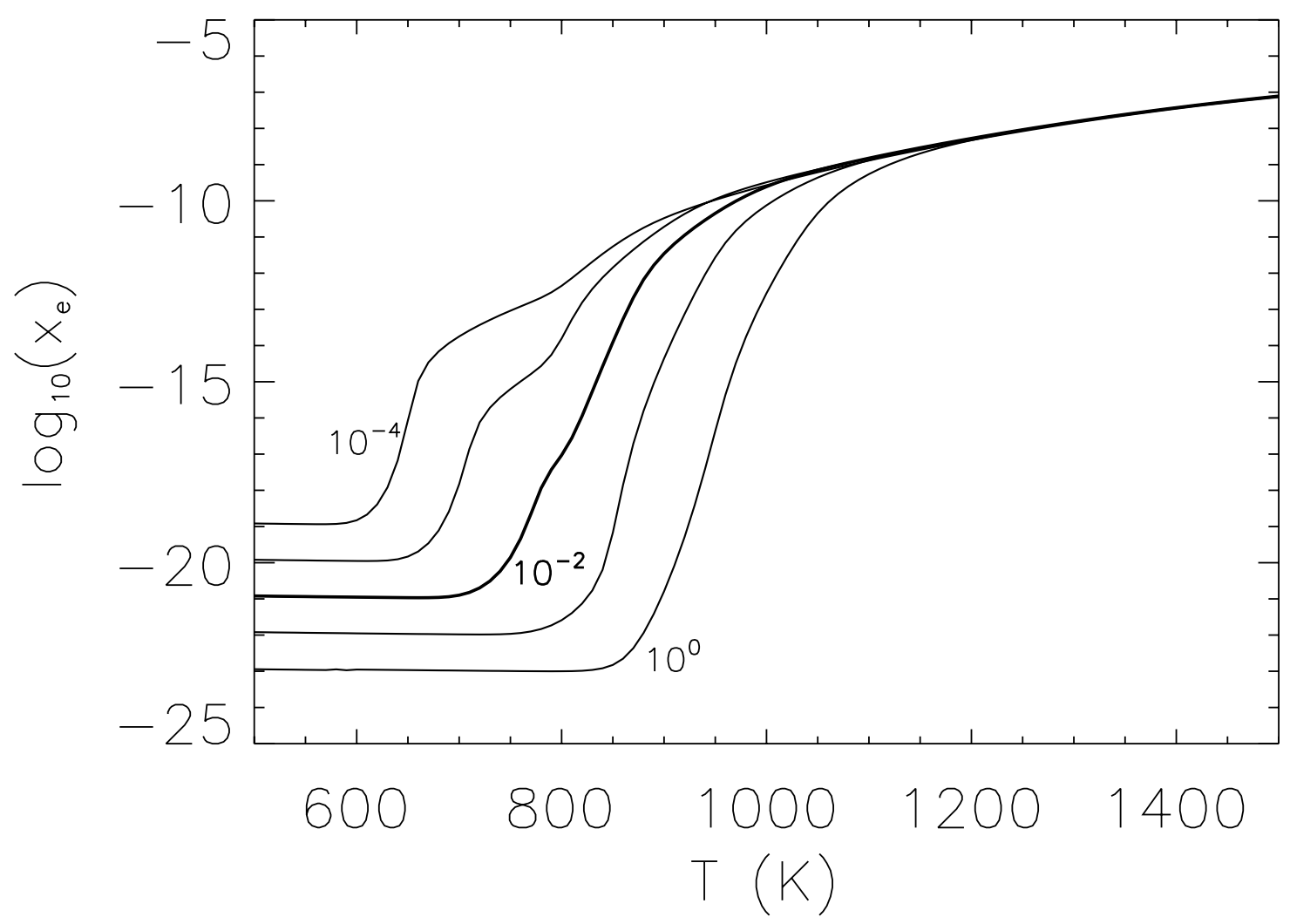

Fig. 12.- Electron fraction vs. temperature for varying dust-to-gas ratios (as labeled). Other parameters are held at their values in the canonical case. At low temperatures ( $\lesssim 600 \mathrm{~K})$, the electron fraction is inversely proportional to the dust-to-gas ratio. At high temperatures ( $\gtrsim 1100 \mathrm{~K}$, the electrons come mostly from thermal ionization and their abundance is independent of the dust-to-gas ratio. At intermediate temperatures, the electron fraction is highly sensitive (but still inversely related) to the dust-to-gas ratio. 


\subsection{Variation with Gas Density}

We now investigate the effects of varying the gas density, keeping other parameters the same as our canonical case $\left(W=5 \mathrm{eV}\right.$, dust-to-gas ratio $\left.10^{-2}\right)$. Figure 13 shows the

electron fraction as a function of temperature for the case of a gas density $n_{\mathrm{H}_{2}}=10^{13} \mathrm{~cm}^{-3}$, an order of magnitude below the canonical value. Figure 14 shows the electron fraction as a function of temperature for the case of a gas density $n_{\mathrm{H}_{2}}=10^{16} \mathrm{~cm}^{-3}$, a factor of 100 above the canonical value. Figure 15 shows the average charge on grains as a function of temperature, for various gas densities. The same trends are apparent: at low temperatures ( $\lesssim 700 \mathrm{~K}$ ), grains are nearly neutral, becoming very negatively charged at temperatures of about 800 to $1000 \mathrm{~K}$, then becoming less negatively charged at higher temperatures. For higher gas densities, these transitions occur at slightly higher temperatures; e.g., a ten-fold increase in $n_{\mathrm{H}_{2}}$ shifts the transitions higher in temperature by only about $60 \mathrm{~K}$. Likewise, Figure 16 shows the electron fraction as a function of gas density. Again it is seen that the charging is relatively insensitive to the gas density. 


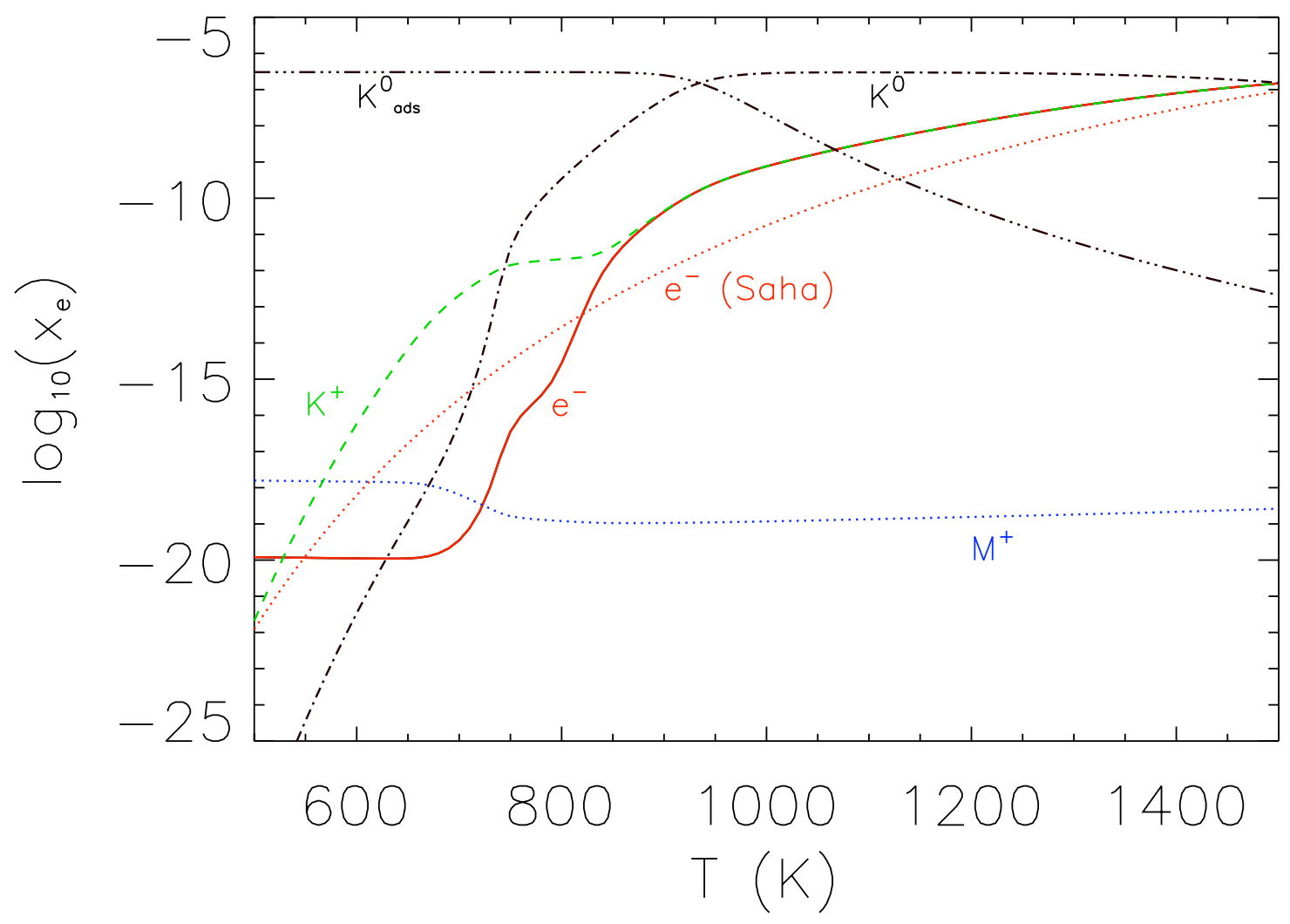

Fig. 13.- Abundances of electrons (solid curve), atomic (Mg) ions (dotted curve), $\mathrm{K}^{+}$ions (dashed curve), gas-phase $\mathrm{K}^{0}$ atoms (dash-dot curve), and bound $\mathrm{K}$ atoms (dash-dot-dot-dot curve), as functions of temperature $T$, for the case with canonical parameters (see Figure 2 ), but with gas density $10^{13} \mathrm{~cm}^{-3}$. The electron fraction predicted using the Saha equation (red dotted curve) is shown for comparison. 


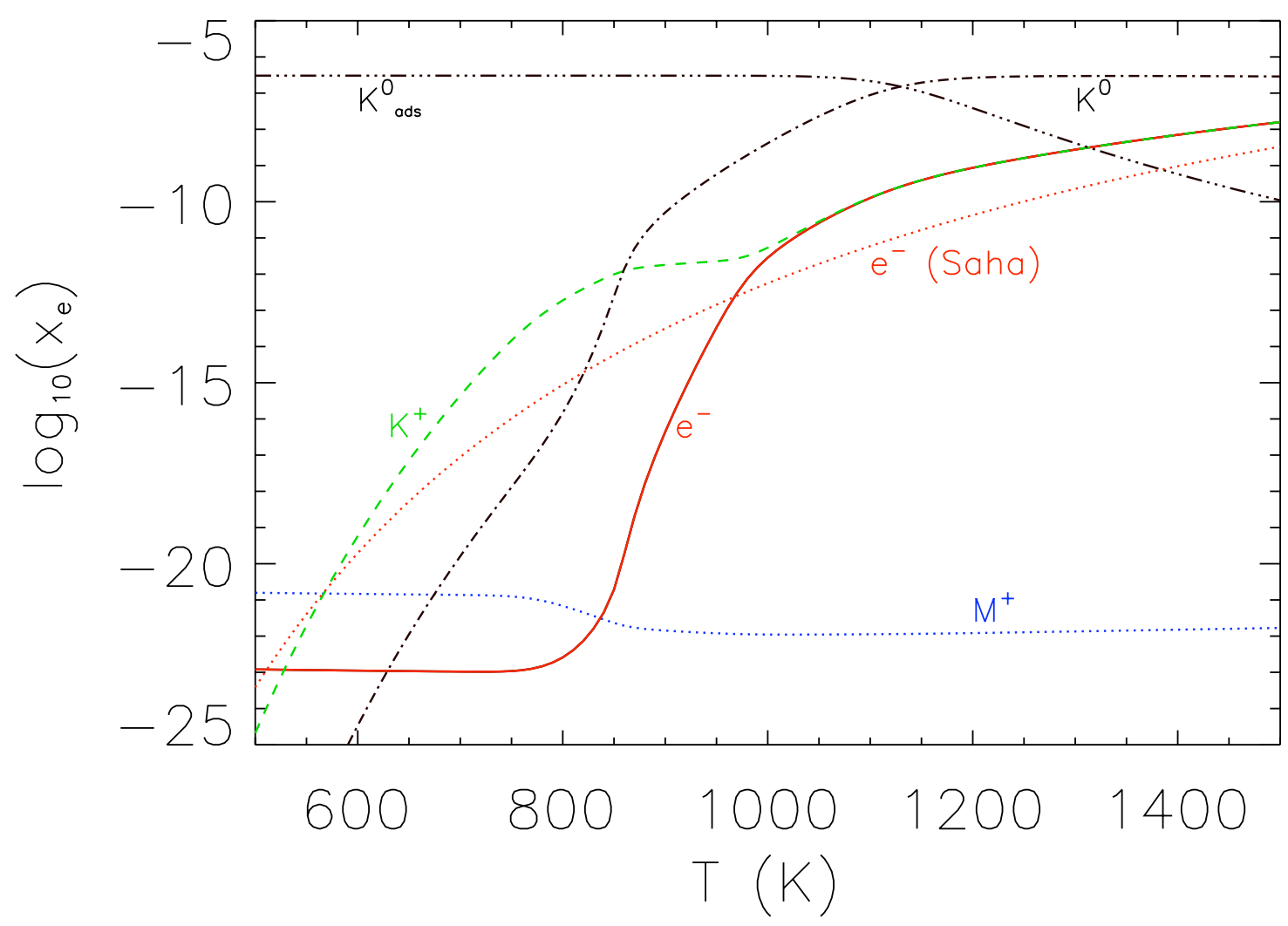

Fig. 14.- Abundances of electrons (solid curve), atomic (Mg) ions (dotted curve), $\mathrm{K}^{+}$ions (dashed curve), gas-phase $\mathrm{K}^{0}$ atoms (dash-dot curve), and bound $\mathrm{K}$ atoms (dash-dot-dot-dot curve), as functions of temperature $T$, for the case with canonical parameters (see Figure 2), but with gas density $10^{16} \mathrm{~cm}^{-3}$. 


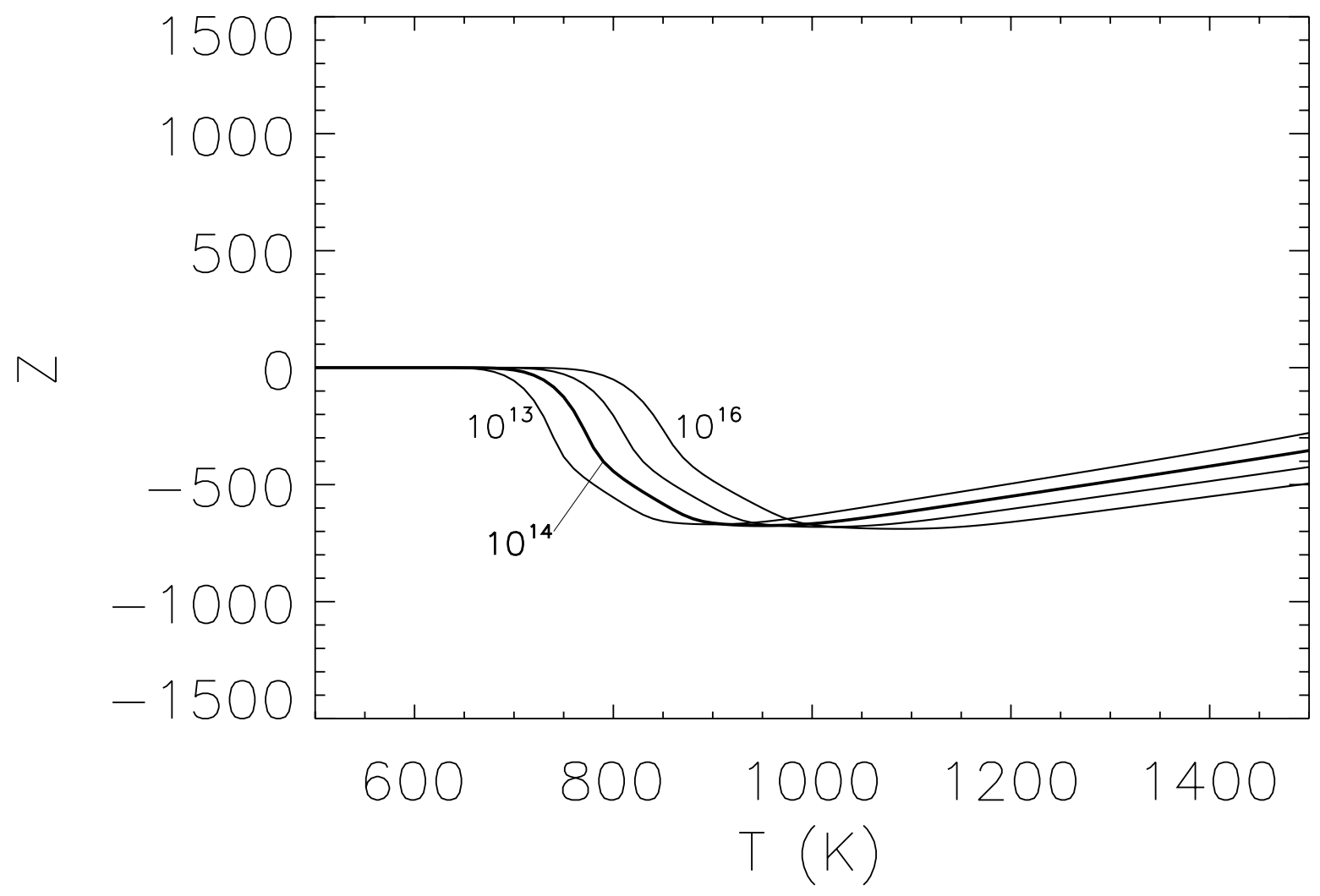

Fig. 15.- Charge on dust grains as a function of temperature, for our canonical values (see Figure 2), but for various values of $n_{\mathrm{H}_{2}}$.

\section{Application to Disks}

\subsection{Inner Edge of MRI Dead Zone}

Our chemical network allows us to calculate ionization levels in dusty plasmas at high temperatures. An important application is to determining the inner edge of the MRI dead 


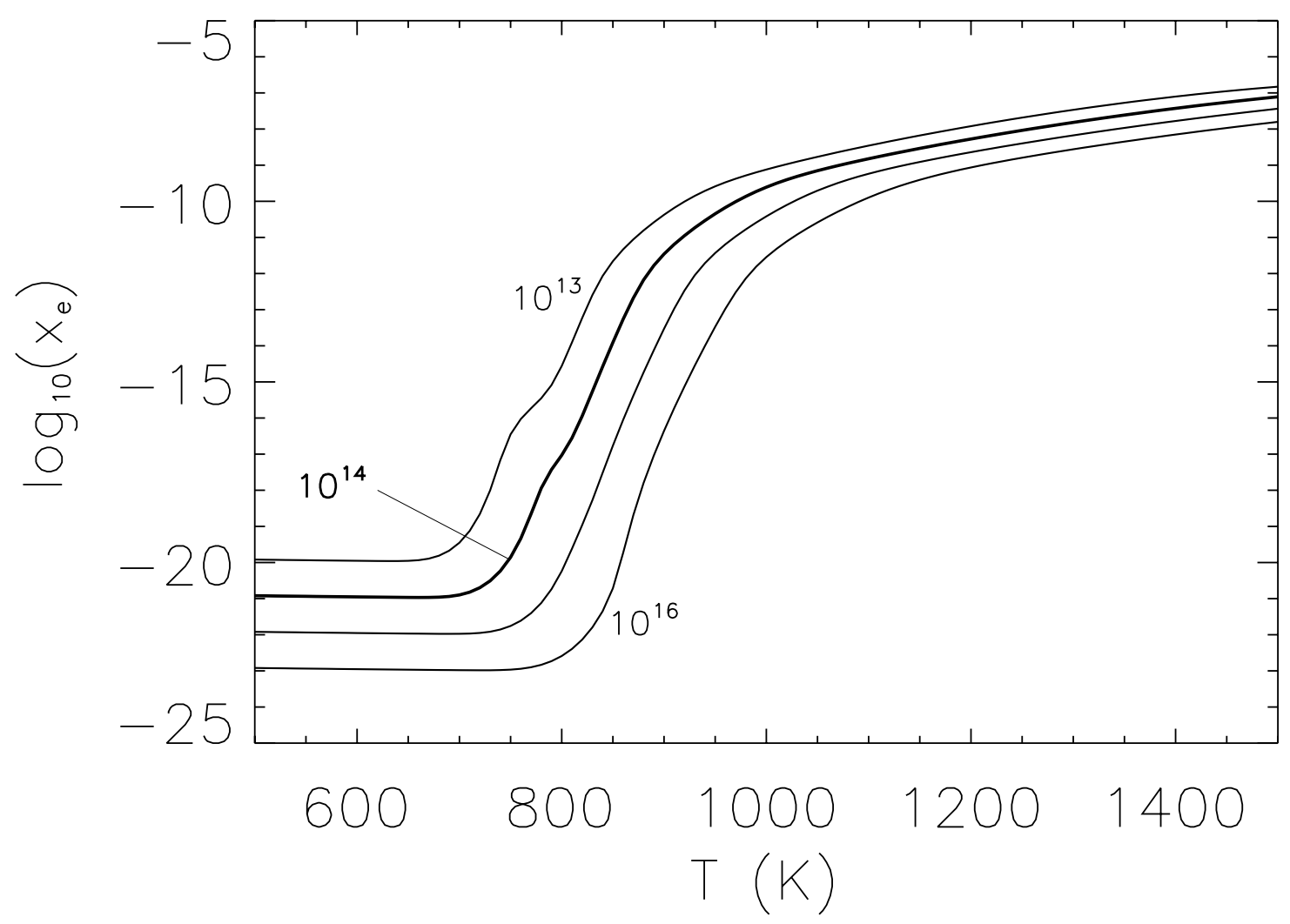

Fig. 16.- Electron fraction as a function of temperature, for our canonical values (see Figure 2), but for various values of $n_{\mathrm{H}_{2}}$.

zone, that region of the disk where the MRI cannot lead to turbulence at the midplane. Jin (1996) and Gammie (1996) both realized that ionization levels at PPD midplanes are too low to permit good coupling of the gas to the magnetic fields, at least in regions where the temperatures are below about $1000 \mathrm{~K}$. Closer to the Sun, at heliocentric distances inside roughly 0.1 to $1 \mathrm{AU}$, temperatures may exceed this threshold, and they argued that thermal ionization of alkali elements in the gas would lead to ionization levels high enough to permit the MRI. Because the mass flow changes abruptly at the dead zone edge, the 
location of the transition bears significantly on the disk's structure and the interpretation of its spectral energy distribution (e.g., Hasegawa \& Pudritz 2010; Zhu et al. 2010).

Using our chemical network, we identify precisely what temperatures are required for sufficient coupling. In Figures 17, 19 we plot the Elsasser numbers associated with Ohmic dissipation $\left(\Lambda_{\mathrm{O}}\right)$, ambipolar diffusion $\left(\Lambda_{\mathrm{A}}\right)$ and the Hall effect $\left(\Lambda_{\mathrm{H}}\right)$. The Elsasser number $v_{A}^{2} /(\eta \Omega)$ is the time in orbits for magnetic fields to diffuse across the fastest-growing MRI wavelength; $v_{\mathrm{A}}=B /(4 \pi \rho)^{1 / 2}$ is the Alfven speed, $\Omega$ is the Keplerian orbital frequency, and $\eta$ is the relevant diffusivity. The Ohmic, ambipolar and Hall diffusivities are straightforward to compute from the charged species' abundances, gyrofrequencies around the magnetic field of strength $B$, and rates of collision with other species (Desch 2004; Wardle 2007). In Figures 17, 19 we plot the three Elsasser numbers as functions of temperature $T$, for various combinations of heliocentric distance $r$, gas density $n_{\mathrm{H} 2}$, and magnetic field strength, set by fixing the plasma beta parameter $\beta=P_{\text {gas }} /\left(B^{2} / 8 \pi\right)$. MRI turbulence is switched off by an Ohmic Elsasser number $\Lambda_{O}<1$ (Sano \& Inutsuka 2001, Turner et al. 2007) or an ambipolar Elsasser number $\Lambda_{A}<1$ (Bai \& Stone 2011), while Hall Elsasser numbers $\Lambda_{H} \ll 1$ replace the turbulence with a laminar flow in which magnetic torques nevertheless yield rapid angular momentum transport (Kunz \& Lesur 2013; Lesur et al. 2014).

We assume a $1 M_{\odot}$ star in what follows. Figure 17 pertains to conditions at 0.1 AU. The top panel has $n_{\mathrm{H} 2}=10^{11} \mathrm{~cm}^{-3}$ and $B \sim 1 \mathrm{G}$, appropriate for the disk atmosphere well above the midplane, and the bottom panel has $n_{\mathrm{H} 2}=10^{14} \mathrm{~cm}^{-3}$ and $B \sim 1 \mathrm{G}$, appropriate for the midplane. As ionization levels increase with increasing temperature, the Elsasser numbers also generally increase. For the disk atmosphere, the last quantity to do so is clearly the one associated with ambipolar diffusion, which therefore is the dominant mechanism suppressing the MRI and setting the location of the dead zone inner edge. In the disk atmosphere at $0.1 \mathrm{AU}$, temperatures must exceed $1150 \mathrm{~K}$ for the MRI to operate. 
At the midplane, ignoring the complications introduced by the Hall effect, ambipolar and Ohmic diffusion are equally important for setting the dead zone inner edge, which will lie where temperatures exceed about $950 \mathrm{~K}$.

Figure 18 pertains to conditions at $1 \mathrm{AU}$. The top panel has $n_{\mathrm{H} 2}=10^{8} \mathrm{~cm}^{-3}$ and $B \sim 10^{-2} \mathrm{G}$, appropriate for the disk atmosphere, and the bottom panel has $n_{\mathrm{H} 2}=10^{11} \mathrm{~cm}^{-3}$ and $B \sim 10^{-2} \mathrm{G}$, appropriate for the midplane. In the atmosphere, ambipolar diffusion is again seen to be the main process suppressing the MRI, and in the range of temperatures we consider, the MRI is never allowed. At the midplane, ambipolar diffusion once more dominates, and initiating the MRI requires temperatures above about $900 \mathrm{~K}$.

Finally, Figure 19 pertains to conditions at 0.1 AU, but during an epoch of increased density, such as might occur around the onset of an FU Orionis accretion outburst. The top panel has $n_{\mathrm{H} 2}=10^{13} \mathrm{~cm}^{-3}$ and $B \sim 10 \mathrm{G}$, appropriate for the disk atmosphere, and the bottom panel has $n_{\mathrm{H} 2}=10^{16} \mathrm{~cm}^{-3}$ and $B \sim 10 \mathrm{G}$, appropriate for the midplane. In the atmosphere, ambipolar diffusion is again the dominant process suppressing the MRI; the Hall term is comparable. Temperatures must exceed about $950 \mathrm{~K}$ for the MRI to operate. At the midplane, Ohmic dissipation and the Hall effect are of similar strengths, and temperatures must exceed about $1070 \mathrm{~K}$ for the MRI to operate.

If these examples are representative, then it would seem that the dead zone's inner edge is set by the action of ambipolar diffusion, not Ohmic dissipation. In other respects, however, our results are similar to those that have been found using the Saha equation. In Figures 17, 19 we also plot the Ohmic Elsasser number under gas-phase collisional ionization of potassium, with the resistivity obtained from the standard formula $\eta=234 T^{1 / 2} x_{\mathrm{e}}^{-1}$ (Blaes \& Balbus 1994; Equation 41). Our chemical network with thermionic and ion emission yields Ohmic resistivities lower, and Elsasser numbers higher, by an order of magnitude or more at temperatures near 1000 K. For the range of conditions we consider, 
initiating the MRI in disk atmospheres or midplanes generally requires temperatures of at least 900 to $1150 \mathrm{~K}$, remarkably similar to the generally assumed value of about $1000 \mathrm{~K}$.

\subsection{Stellar Magnetospheric Loading}

Young stars grow by accreting material from their disks. The gas flows to the star along the stellar magnetic field lines if the mass accretion rate is too low for the incoming gas to crush the stellar magnetosphere (Edwards et al. 1994; Bouvier et al. 2007). The gas could be loaded onto the stellar field either through the field lines' diffusion into the disk (Miller \& Stone 1997), through magnetic reconnection (Hirose et al. 1997), or through a

Rayleigh-Taylor type instability (Romanova et al. 2008). Which scenario predominates, and how quickly the mass is loaded by each of the three mechanisms, depends on the magnetic diffusivity. We suggest that the transition from loading by dynamical instability to loading by magnetic diffusion occurs when the diffusivity exceeds $h^{2} \Omega$, where $h$ is the disk density scale height. This is based on the finding from ideal-MHD modeling that the plasma beta is about unity at the magnetosphere's edge where loading occurs (Romanova et al. 2012). Consequently the scale on which magnetic and Coriolis forces balance, and fastest-growing vertical wavelength of the magnetorotational instability, will be about $h$.

The mass loading takes place not too far from the co-rotation radius, where the Keplerian orbital period matches the star's rotation period. Accreting $\mathrm{T}$ Tauri stars typically rotate about once a week (Bouvier et al. 1993), so the co-rotation radius around a $1 M_{\odot}$ star is at $r \approx 0.07 \mathrm{AU}$. In Figure 20 we present results near the co-rotation point for the Ohmic diffusivity $\eta_{\mathrm{O}}$, Hall diffusivity $\eta_{\mathrm{H}}$, and ambipolar diffusivity $\eta_{\mathrm{A}}$, as functions of temperature. Three combinations of density and plasma beta parameter $\beta=P_{\mathrm{g}} / P_{\mathrm{mag}}$ are shown: $n_{\mathrm{H} 2}=10^{12} \mathrm{~cm}^{-3}, \beta=1 ; n_{\mathrm{H} 2}=10^{14} \mathrm{~cm}^{-3}, \beta=1$; and $n_{\mathrm{H} 2}=10^{16} \mathrm{~cm}^{-3}, \beta=100$. We choose $\beta=1$ for the first two panels, yielding a local field strength $B$ that increases 
slightly with temperature. In the third panel we select $\beta=100$ since the stellar magnetic field at $0.07 \mathrm{AU}$ is unlikely to exceed ten gauss, given a few kilogauss value at the stellar photosphere and a dipolar falloff with distance. The third panel's parameters are thus more likely to be found in the higher accretion rate regime where the inflowing gas has enough ram pressure to force the stellar fields inward.

Grey shading in Figure 20 indicates diffusivities high enough that diffusive loading is likely to dominate. The transition from dynamical to diffusive loading takes place at a temperature in the range from $960 \mathrm{~K}\left(\right.$ at $\left.n_{\mathrm{H} 2}=10^{14} \mathrm{~cm}^{-3}\right)$ to $1080 \mathrm{~K}\left(\right.$ at $\left.n_{\mathrm{H} 2}=10^{12} \mathrm{~cm}^{-3}\right)$. Ambipolar diffusion causes the transition except in the case with the highest density and weakest magnetic field, where Ohmic diffusion is stronger. The Hall term is also signficant near the threshold in all three cases. Temperatures below the transition are quite plausible if accretion heating is weak. The thresholds are comparable to the $1060 \mathrm{~K}$ equilibrium temperature of a blackbody directly exposed to the starlight at 0.07 AU. Furthermore corotation lies beyond the dust sublimation front, so starlight can be absorbed before reaching $0.07 \mathrm{AU}$. In a passively heated protoplanetary disk, temperatures at $0.07 \mathrm{AU}$ are predicted to be $<690 \mathrm{~K}$ (Lesniak \& Desch 2011). We conjecture that the stellar magnetic field may diffuse slowly into the hot gas nearer the star, but field lines that escape this region - either through reconnection or disruption of the disk's inner edge by a dynamical instability - can quickly diffuse further into the disk and load gas from the disk on the stellar magnetosphere.

Two points seem worth emphasizing. First, the close match between the diffusive loading threshold and the temperatures expected in passively heated disks means that the loading mechanism can switch depending on conditions. Second, at temperatures near the threshold, thermionic and ion emission reduce the diffusivity by orders of magnitude compared with the Saha equation. The grains' work function thus likely impacts the 
character of diffusive loading on young stars' magnetospheres. These effects should be included in future studies.

\subsection{Current Sheet Instabilities}

The treatment of high-temperature ionization in protoplanetary disks also can be applied to the "short-circuit" instability of current sheets. In protoplanetary disks with magnetorotational turbulence, it is reasonable to assume (and simulations show) that a commonly occurring magnetic geometry has one region of more-or-less uniform magnetic field adjacent to another region with the field lines oriented in the opposite direction (Sano et al. 2007; Hubbard et al. 2012). The surface separating the two is marked by steep magnetic gradients and high current densities, and is termed the current sheet. Ohmic dissipation can lead to heating in this sheet. Hubbard et al. (2012) argued that if the gas' magnetic diffusivity $\eta$ decreases with increasing temperature then the heating can grow exponentially: increased temperature leads to lower diffusivity; the current concentrates in the lower-diffusivity layer, accompanied by more Ohmic heating; so the temperature increases further. The runaway heating can be quite rapid, potentially taking hours or less, depending on the ionization properties of the gas. Hubbard et al. (2012) and McNally et al. (2013) further argued that this sudden heating might be the mechanism behind the formation of chondrules, which are known to have been melted suddenly by some "flash-heating" process, then cooled and crystallized over the course of hours (Connolly et al. 2006). Whether current sheet instabilities can take place in protoplanetary disks, and whether they can grow fast enough to be the chondrule formation mechanism, are key questions that hinge on the ionization state of the gas at high temperatures.

The growth rate of the short-circuit instability was calculated by Hubbard et al. (2012), who performed a linear analysis on a background state in which the magnetic field increases 
linearly from $-B_{0} / 2$ to $+B_{0} / 2$ as $x$ runs from $-L / 2$ to $+L / 2$, where $x$ is the direction normal to the current sheet. They assumed that perturbations in the magnetic field, current density, etc., vary as $\exp (+\lambda t+i k x)$, where $k$ is the wavenumber of the perturbation and $\lambda$ is the growth rate. The growth rate is then found to satisfy the equation

$$
\lambda^{2}+\lambda\left(t_{\text {cool }}^{-1}+t_{\text {diff }}^{-1}+t_{\eta}^{-1}\right)+t_{\text {diff }}^{-1}\left(t_{\text {cool }}^{-1}-t_{\eta}^{-1}\right)=0
$$

where $t_{\text {cool }}$ is the timescale over which emission of radiation cools the region; we specify its value below. Here $t_{\text {diff }}=1 /\left(k^{2} \eta_{0}\right)$ is the timescale for the magnetic field to diffuse one wavelength, and $\eta_{0}$ is the magnetic diffusivity in the background state. Other zero subscripts likewise indicate conditions in the background state. The timescale $t_{\eta}$ can be defined as

$$
t_{\eta}=-\left(\frac{\partial \ln \eta}{\partial \ln T}\right)_{T=T_{0}}^{-1} t_{\text {heat }}
$$

where

$$
t_{\text {heat }}=\frac{4 \pi n_{\mathrm{n}} k_{\mathrm{B}} T_{0}}{(\gamma-1) B_{0}^{2}} \frac{L^{2}}{\eta_{0}}=\frac{\beta_{0}}{2(\gamma-1)} \frac{L^{2}}{\eta_{0}},
$$

is the timescale for Ohmic dissipation to heat the gas significantly. We have written $t_{\text {heat }}$ in terms of the plasma beta parameter in the zero-order state, $\beta_{0}=P_{0} /\left(B_{0}^{2} / 8 \pi\right)$, which makes clear that the heating rate is directly tied to the rate at which magnetic field lines diffuse into the current sheet. In the usual case where $\eta$ decreases with increasing temperature, $t_{\eta}>0$. In that case, unstable growth is assured if $t_{\eta}<t_{\text {cool }}$.

To determine $t_{\text {cool }}$ we imagine a slab of thickness $L$, heated to temperature $T$ by Ohmic dissipation. The slab will cool as it emits radiation that must diffuse through the surrounding gas. If this gas has density $\rho$, heat capacity $C_{\mathrm{P}}$, and opacity (per mass of gas) $\kappa$, the appropriate timescale is the radiative diffusion time,

$$
t_{\mathrm{rd}}=\frac{3}{64 \pi^{2}} \frac{\rho^{2} C_{\mathrm{P}} \kappa}{\sigma T^{3}} L^{2}
$$

(Mihalas \& Mihalas 1984; Kippenhahn \& Weigert 1990; Desch \& Connolly 2002). So that the scenario is relevant to chondrule formation, we adopt $\rho=1 \times 10^{-9} \mathrm{~g} \mathrm{~cm}^{-3}, T=1500 \mathrm{~K}$, 
and $C_{\mathrm{P}}=8 \times 10^{7} \mathrm{erg} \mathrm{g}^{-1} \mathrm{~K}^{-1}$. As for the opacity, a reasonable value would be $26 \mathrm{~cm}^{2} \mathrm{~g}^{-1}$ if dust were present (at $T=1500 \mathrm{~K}$ ), but since micron-sized dust generally vaporizes within minutes in the chondrule-forming region, a more reasonable opacity would be that of chondrules alone, $\kappa \approx 0.03 \mathrm{~cm}^{2} \mathrm{~g}^{-1}$ (Morris \& Desch 2010). We find $t_{\mathrm{rd}}=5200\left(L / 10^{5} \mathrm{~km}\right)^{2} \mathrm{~s}$ if $\kappa=26 \mathrm{~cm}^{2} \mathrm{~g}^{-1}$, and $t_{\mathrm{rd}}=6\left(L / 10^{5} \mathrm{~km}\right)^{2} \mathrm{~s}$ if $\kappa=0.03 \mathrm{~cm}^{2} \mathrm{~g}^{-1}$. Note that McNally et al. (2013) took densities $\rho \sim 10^{-7} \mathrm{~g} \mathrm{~cm}^{-3}$, which would make cooling slower by 4 orders of magnitude; but even in a disk with 10 times more material than the minimum mass solar nebula (Weidenschilling 1977), these densities are only achieved far inside 1 AU, so some outward transport process would be needed to place the chondrules in the asteroid belt. At the lower densities that seem more likely to prevail, the current sheet cools by radiative diffusion in a $t_{\text {cool }}$ of seconds to hours.

With the cooling timescale in hand, it is simple to find the ratio of $t_{\eta}$ and $t_{\text {cool }}$ :

$$
\frac{t_{\eta}}{t_{\text {cool }}}=-\left(\frac{\partial \ln \eta}{\partial \ln T}\right)^{-1} \frac{\beta_{0}}{2(\gamma-1) \eta_{0}} \frac{64 \pi^{2}}{3} \frac{\sigma T^{3}}{\rho^{2} C_{\mathrm{P}} \kappa}
$$

Note that the ratio does not depend on the size of the slab: the heating timescale relies on diffusion of magnetic flux, and the cooling timescale relies on diffusion of radiation, so both scale as $L^{2}$. Unstable modes require this ratio to be $<1$, or

$$
\eta>\eta_{\min }=\left|\frac{\partial \ln \eta}{\partial \ln T}\right|^{-1} \frac{\beta_{0}}{2(\gamma-1)} \frac{64 \pi^{2}}{3} \frac{\sigma T^{3}}{\rho^{2} C_{\mathrm{P}} \kappa} .
$$

For reasonable parameters $\left(\rho=10^{-9} \mathrm{~g} \mathrm{~cm}^{-3}, T=1500 \mathrm{~K}, \gamma=7 / 5, C_{\mathrm{P}}=8 \times 10^{7} \mathrm{erg} \mathrm{g}^{-1} \mathrm{~K}^{-1}\right)$, and assuming $\beta_{0}=10$ and $\kappa=26 \mathrm{~cm}^{2} \mathrm{~g}^{-1}, \eta_{\min } \approx 2.4 \times 10^{17}|\partial \ln \eta / \partial \ln T|^{-1} \mathrm{~cm}^{2} \mathrm{~s}^{-1}$. (or $\sim 10^{3}$ times higher if dust evaporates). The resistivity must be high enough that the current sheet can be continually fed with magnetic field lines by diffusion, faster than radiation can cool the gas. We can also express this requirement as a maximum electron fraction $x_{\mathrm{e}}$, using the following relationship:

$$
\eta=9060\left(\frac{T}{1500 \mathrm{~K}}\right)^{1 / 2} x_{\mathrm{e}}^{-1} \mathrm{~cm}^{2} \mathrm{~s}^{-1}
$$


(Hayashi 1981; Balbus \& Terquem 2001). We find the unstable modes require $x_{\mathrm{e}}<x_{\mathrm{e}, \max } \approx 3.7 \times 10^{-14}|\partial \ln \eta / \partial \ln T|$, or three orders of magnitude smaller if dust evaporates.

For our canonical case depicted in Figure 2, if $T<750 \mathrm{~K}$ there is no variation in the electron density with temperature, in which case $x_{\mathrm{e}, \max } \approx 0$, and no unstable modes can be expected. For $T>750 \mathrm{~K},|\partial \ln \eta / \partial \ln T| \approx 100$ (the electron density rises 8 orders of magnitude as $T$ increases from $750 \mathrm{~K}$ to $900 \mathrm{~K}$ ), so $x_{\mathrm{e}, \max } \approx 3.7 \times 10^{-12}$ (or three orders of magnitude smaller if dust evaporates). This means the instability can proceed until $T \approx 900 \mathrm{~K}$. For hotter temperatures the electron density is too high for field lines to diffuse into the current sheet fast enough to overcome the radiative cooling. So, for the short-circuit instability to act, the ambient temperature of gas surrounding the current sheet must fall in the narrow range $750 \mathrm{~K} \lesssim T \lesssim 900 \mathrm{~K}$. The case with grains with work function $W=6 \mathrm{eV}$ is similar to the canonical case. For the $W=2 \mathrm{eV}$ case, $|\partial \ln \eta / \partial \ln T| \approx 14$ for $T$ between 600 and $800 \mathrm{~K}$. We then find $x_{\mathrm{e}, \max } \approx 5 \times 10^{-13}$ or so. A wider range of temperatures, extending down to $650 \mathrm{~K}$, is then allowed to be unstable. Again, if dust evaporates in the chondrule formation region, then the faster radiative cooling means the maximum allowable electron density is much lower, by about three orders of magnitude.

A second requirement for the current sheet instability is that a current sheet can be set up in the first place. This in turn requires the existence of magnetic turbulence in the disk, almost certainly from the magnetorotational instability (MRI). The MRI is suppressed if the Ohmic Elsasser number $\Lambda_{\mathrm{O}}=\left(v_{\mathrm{A}}^{2} / \Omega\right) / \eta$ is less than unity, or if $\eta \gtrsim v_{\mathrm{A}}^{2} / \Omega$, where $v_{\mathrm{A}}^{2}$ is the Alfven number and $\Omega$ the Keplerian orbital frequency (Jin 1996). From the definition of the plasma beta number, $v_{\mathrm{A}}^{2}=(2 / \beta) C^{2}$, where $C$ is the sound speed; in disks in which the MRI has saturated, typically $\beta \sim 10^{2}$ (Miller \& Stone 2000; Hirose et al. 2006). Therefore the existence of the MRI requires $\eta \lesssim 0.02 C^{2} / \Omega$. Note that the existence of a current sheet 
demands a low value of the magnetic diffusivity, while the short-circuit instability requires a high value of $\eta$. For reasonable parameters (say, $T \approx 800 \mathrm{~K}$, at $1 \mathrm{AU}$ in the disk), the MRI requires $\eta \lesssim 2.8 \times 10^{15} \mathrm{~cm}^{2} \mathrm{~s}^{-1}$, or $x_{\mathrm{e}} \gtrsim 2.5 \times 10^{-12}$. For our canonical case, there is an exceedingly narrow range of electron fractions in the ambient nebula that can both lead to current sheets and the short-circuit instability: $2.5 \times 10^{-12} \lesssim x_{\mathrm{e}} \lesssim 3.8 \times 10^{-12}$, corresponding to a very narrow temperature range about $10 \mathrm{~K}$ wide, centered on about $900 \mathrm{~K}$. As the work function of solids is reduced, the range narrows. For the $W=2 \mathrm{eV}$ case, there is in fact no overlap: the electron fractions needed for runaway growth are not compatible with the operation of the MRI and the existence of current sheets. If dust evaporates in the chondrule formation region, there is no overlap in conditions for any value of $W$. The net effect of all these factors is that the gas ionization needed to generate magnetic turbulence and to initiate the short-circuit instability are unlikely to be simultaneously met in protoplanetary disks.

Finally, we point out one additional problem for the short-circuit instability, which is its need to grow faster than the chemical timescales will allow. The timescales in the problem must be $<t_{\text {cool }}$, which we argue is no more than $5000 \mathrm{~s}$ (for $\rho=10^{-9} \mathrm{~g} \mathrm{~cm}^{-3}$ and no evaporation of dust). The instability requires the gas resistivity to decrease as the temperature increases, on these timescales. The steady-state electron abundance indeed increases with temperature, but the ionization chemistry takes time to reach steady state. According to Figures 2 and 5, the electron abundance starts to rise with temperature above $750 \mathrm{~K}$ or so, but the chemical timescales to achieve these higher electron abundances also rise. Above about $800 \mathrm{~K}$, the chemical timescales exceed $5000 \mathrm{~s}$ and approach $10^{5} \mathrm{~s}$. This effectively reduces $|\partial \ln \eta / \partial \ln T|$, potentially by 1 to 2 orders of magnitude, making the instability more difficult to initiate and grow.

In summary, the short-circuit instability requires a rise in electron abundance with 
temperature, together with an initially large magnetic diffusivity so that magnetic heating is faster than radiative cooling. However, generating current sheets in the first place requires a small magnetic diffusivity. Depending on such parameters as gas density, opacity, grain work function, etc., there is either no way to simultaneously satisfy these requirements, or they are satisfied only in a narrow (perhaps $10 \mathrm{~K}$ ) temperature range, typically above $700 \mathrm{~K}$. The short-circuit instability therefore appears to require rather special conditions. Moreover, the requirement that the temperature start above $700 \mathrm{~K}$ is inconsistent with the presence of primary sulfur in chondrules, which indicates ambient temperatures $<650 \mathrm{~K}$ (Lodders 2003). Even if short-circuit instabilities can arise from current sheets, they do not seem compatible with the chondrule formation mechanism.

\section{Conclusions}

The ionization state of the gas hotter than about $500 \mathrm{~K}$ determines how well protostellar disks couple to their internal magnetic fields and to the fields of the central star. Properly calculating the ionization state is especially important for assessing where the magnetorotational instability can operate, which bears on the question of how the disks evolve over time. It bears on whether disk gas can be loaded onto a star's magnetic field. It also bears on whether current sheets can dissipate magnetic energy via the current sheet instability. Previous treatments of ionization at high temperatures have assumed that potassium atoms are in the gas phase and that they are ionized according to the Saha equation. This approach ignores the role of dust and the fact that ions and electrons strike grains far more often than they meet one another in the gas and recombine. Furthermore gas-phase collisions are not the only ionization process. At high temperatures, the grains also emit electrons and ions into the gas. Balancing thermionic (and ion) emission against adsorption of electrons (and ions) on the grains yields the Saha-Langmuir equation, which 
is similar in form to the Saha equation, but instead of the first ionization potential of the gas-phase atoms (e.g. $4.34 \mathrm{eV}$ for potassium) involves the work function of the grain material. Work functions typical of the solid materials in protoplanetary disks are near $5 \mathrm{eV}$ (silicates at 5.0 to $5.4 \mathrm{eV}, \mathrm{FeNi}$ metal at $4.4 \mathrm{eV}$, graphite $4.62 \mathrm{eV}$ ). These exceed the first ionization potential of potassium, but because the grains present so much surface area, the disk gas is more ionized (with higher electron density) than expected based on the Saha equation. These processes are important at temperatures between $700 \mathrm{~K}$ and the dust sublimation threshold. We are the first to identify their role in ionizing the gas in the central regions of protoplanetary disks.

We have incorporated thermionic and ion emission into a chemical network and solved for the steady-state abundances of electrons and other species as functions of temperature and density. Unlike previous analyses, we do not automatically assume that potassium atoms reside in the gas phase; in fact, Lodders (2003) predicts a $50 \%$ condensation temperature of $1006 \mathrm{~K}$, meaning that below about $1000 \mathrm{~K}$, most potassium is locked up in solids. We mimic this effect by using an evaporation energy $\approx 3 \mathrm{eV}$ that potassium atoms must acquire to leave the grain surface. Thus above $800 \mathrm{~K}$, at least one in $10^{4}$ or $10^{5}$ potassium atoms is in the gas phase rather than locked up in the grains. This treatment is approximate, but even if all the sodium were firmly bound to the grains at lower temperatures, cesium atoms are in the gas phase above $850 \mathrm{~K}$ (Lodders 2003) and their abundance is only $10^{4}$ times less than potassium. Some alkali atoms thus occur in the gas below $1000 \mathrm{~K}$.

The most signficant result from our canonical case is a major rise in the electron fraction at temperatures between $700 \mathrm{~K}$ and $900 \mathrm{~K}$. We find that qualitatively, the run of electron fraction with temperature is relatively insensitive to the work function (Figure 8), gas density (Figure 16), and dust-to-gas mass ratio (Figure 12). Quantitatively, 
the temperature at which the electron fraction rises sharply does vary somewhat: if the dust-to-gas mass ratio is increased (decreased) two orders of magnitude, the rise in electron density is shifted about $100 \mathrm{~K}$ to higher (lower) temperatures (Figure 12), with similar results expected for changes in grain size. Likewise, increasing (decreasing) the gas density by two orders of mangitude causes the rise in electron density to occur at temperatures about $100 \mathrm{~K}$ higher (lower) (Figure 16). A notable feature of the results is that the electron density can almost be computed using the Saha equation for potassium, but one must use an ionization potential less than $4.34 \mathrm{eV}$. Above $800 \mathrm{~K}$, for plausible grain work functions, values between 3.5 and $4.0 \mathrm{eV}$ yield the correct ionization state, and that state is significantly more ionized than at lower temperatures. As a robust result, then, thermal ionization can be said to be significant above about $800 \mathrm{~K}$.

A notable feature of the high-temperature regime is that the grains become rather highly charged through directly emitting electrons (or ions). The grain charge can be positive or negative, but for the most likely work function values near $5 \mathrm{eV}$, the grains emit ions and are left with negative charges. The charging is strong above $800 \mathrm{~K}$, and grains of radius $1 \mu \mathrm{m}$ reach charges of about $-700 e$ at $900 \mathrm{~K}$ (Figure 3 ). While much larger than the grain charges found neglecting thermionic and ion emission, this is equivalent to an electric potential of just 1 volt. The charging is strong enough to affect the grains' coagulation at high temperatures. The Coulomb energies that must be overcome for grains to collide are $\sim 10^{3} \mathrm{eV}$, or $\sim 10^{-9} \mathrm{erg}$, so micron-sized grains coming together slower than $10 \mathrm{~cm} \mathrm{~s}^{-1}$ will be mutually repelled by the electrical force. The effects of grain charging on the coagulation of grains have been investigated by Okuzumi (2009). We also point out that if there were any grains composed of materials with work functions $\lesssim 3.5 \mathrm{eV}$, then their positive charges would enhance their collision rates with the negatively charged grains.

We have applied our charging model to the question of the MRI dead zone's inner 
edge. At low temperatures the gas is insufficiently ionized to permit the coupling of the gas to the magnetic field that is fundamental to the MRI. We find that for reasonable disk parameters (gas density, magnetic field strength), the temperature needed for the MRI to operate is set by ambipolar rather than Ohmic diffusion. The typical threshold temperatures are in the range 900 to $1100 \mathrm{~K}$. At the high densities found near the midplane, the thresholds are comparable to or up to $100 \mathrm{~K}$ less than when neglecting the thermionic and ion emission and considering only the Ohmic resistivity. At the lower, atmospheric densities, ambipolar diffusion makes the thresholds up to $300 \mathrm{~K}$ higher than those from the Saha equation and Ohmic resistivity. Nevertheless, the results validate the common belief that thermal ionization requires temperatures above $1000 \mathrm{~K}$. We likewise have investigated the temperatures required to diffusively load the disk mass onto the stellar magnetosphere. We find once again that temperatures below $1000 \mathrm{~K}$ are required, and that the threshold is set by ambipolar diffusion.

The high-temperature ionization also has implications for the onset of the "short-circuit instability" and its role in chondrule formation. This instability relies on the resistivity of the gas decreasing with increasing temperature, so that dissipation of magnetic energy during reconnection events can accelerate. We confirm that above a critical threshold temperature (about $800 \mathrm{~K}$ in our canonical case, but potentially as low as $650 \mathrm{~K}$ ), this condition is met. On the other hand, the presence of primary sulfur (Rubin et al. 1999) in chondrules suggests that they condensed in regions cooler than $650 \mathrm{~K}$, the condensation temperature of sulfur. In addition, we show that there are two extra and seemingly incompatible constraints with which the short-circuit instability must comply. The instability requires the resistivity to change on timescales faster than the gas can cool. This in turn imposes a lower limit on the resistivity, and an upper limit on the electron fraction, so that field lines can diffuse rapidly enough into the reconnection region; typically $x_{\mathrm{e}}$ must not exceed about $10^{-13}$. The short-circuit instability also requires magnetic turbulence to set up the current sheets of 
oppositely oriented field lines. Assuming magnetic turbulence is generated by the MRI, this in turn imposes an upper limit on the resistivity, and a lower limit to the electron fraction; typically $x_{\mathrm{e}}$ must be $10^{-13}$ or greater. For typical conditions, only a small range of electron fractions satisfy both conditions simultaneously, while for many parameters (especially if dust evaporates in the chondrule-forming region), no conditions simultaneously lead to the formation of current sheets and allow the short-circuit instability. This and the constraint that chondrule precursors started at temperatures $<650 \mathrm{~K}$ to retain primary sulfur would seem to imply that current sheets and the short-circuit instability are probably not the chondrule formation mechanism.

We note that thermionic and ion emission may be important to the ionization state of gas in the atmospheres of exoplanets, especially "hot Jupiters". Ohmic dissipation of magnetic energy in their atmospheres has been invoked as the explanation for the inflated radii (above model predictions) of hot Jupiters such as HD209458b and HD189733b (Batygin \& Stevenson 2010; Perna et al. 2010) and may affect atmospheric circulation (Menou 2012; Rogers \& Komacek 2014). At present, the resistivity of the gas in such models is computed essentially using equation 41 (as in Balbus \& Terquem 2001), with the electron density found using the Saha equation for various gas-phase atoms (Batygin \& Stevenson 2010; Rogers \& Komacek 2014). However, hot Jupiters, HD209458b and HD189733b in particular, are predicted to have cloud layers containing condensates such as TiO (Fortney et al. 2010). TiO has a work function of about $4.5 \mathrm{eV}$ (Fomenko 1966; Greiner et al. 2012) and a cosmochemical abundance equivalent to a dust-to-gas ratio $\sim 10^{-5}$ (Lodders 2003). At pressures of 1 bar, and temperatures of $1000 \mathrm{~K}$, the gas density would be $n_{\mathrm{H}_{2}} \approx 6 \times 10^{18} \mathrm{~cm}^{-3}$. We therefore expect that thermionic and ion emission from $\mathrm{TiO}$ particles, and adsorption of electrons and ions onto them, will control the ionization state of the gas, which will then not be described using a Saha equation, above some threshold temperature. Comparing to Figures 12 and 16, we expect that the high density will raise 
the threshold temperature, while the low dust-to-gas ratio would lower the threshold temperature, but that thermionic emission would dominate the charging of the gas above about 800 to 1000 K. Naively, we expect thermionic emission will lead to higher electron fractions and lower Ohmic dissipation than use of the Saha equation alone, but exoplanet atmospheres are far enough out of the regime considered in this paper that other details may play a role. In any case, we recommend that this charging mechanism be considered when calculating Ohmic dissipation in the atmospheres of hot Jupiters.

We also recommend that future effort be directed to measuring the work functions of astrophysically relevant materials, especially those found in protoplanetary disks and exoplanet atmospheres. We culled information from a variety of decades-old sources, but a dedicated effort should be made to consistently compare thermionic and ion emission from silicates, FeNi, graphite particles, and other astrophysical solids.

Where solids exist, they can catalyze the recombinations of electrons and ions on their surfaces, rendering the Saha equation incorrect. But they also emit electrons or ions directly into the gas, at rates that depend on their material properties. In the end, the ionization fraction increases faster with temperature than under the Saha equation. For relevant conditions, we find that the ionization rises significantly above $800 \mathrm{~K}$.

We thank the organizers of the August 2014 meeting "Non-ideal magnetohydrodynamics, stability, and dissipation in protoplanetary disks" at the Niels Bohr International Academy in Copenhagen, for providing a forum for our collaboration. This research was carried out in part at the Jet Propulsion Laboratory, California Institute of Technology, under a contract with the National Aeronautics and Space Administration, and with the support of grant number 13-OSS13-0114 from the Solar Systems Origins program. 


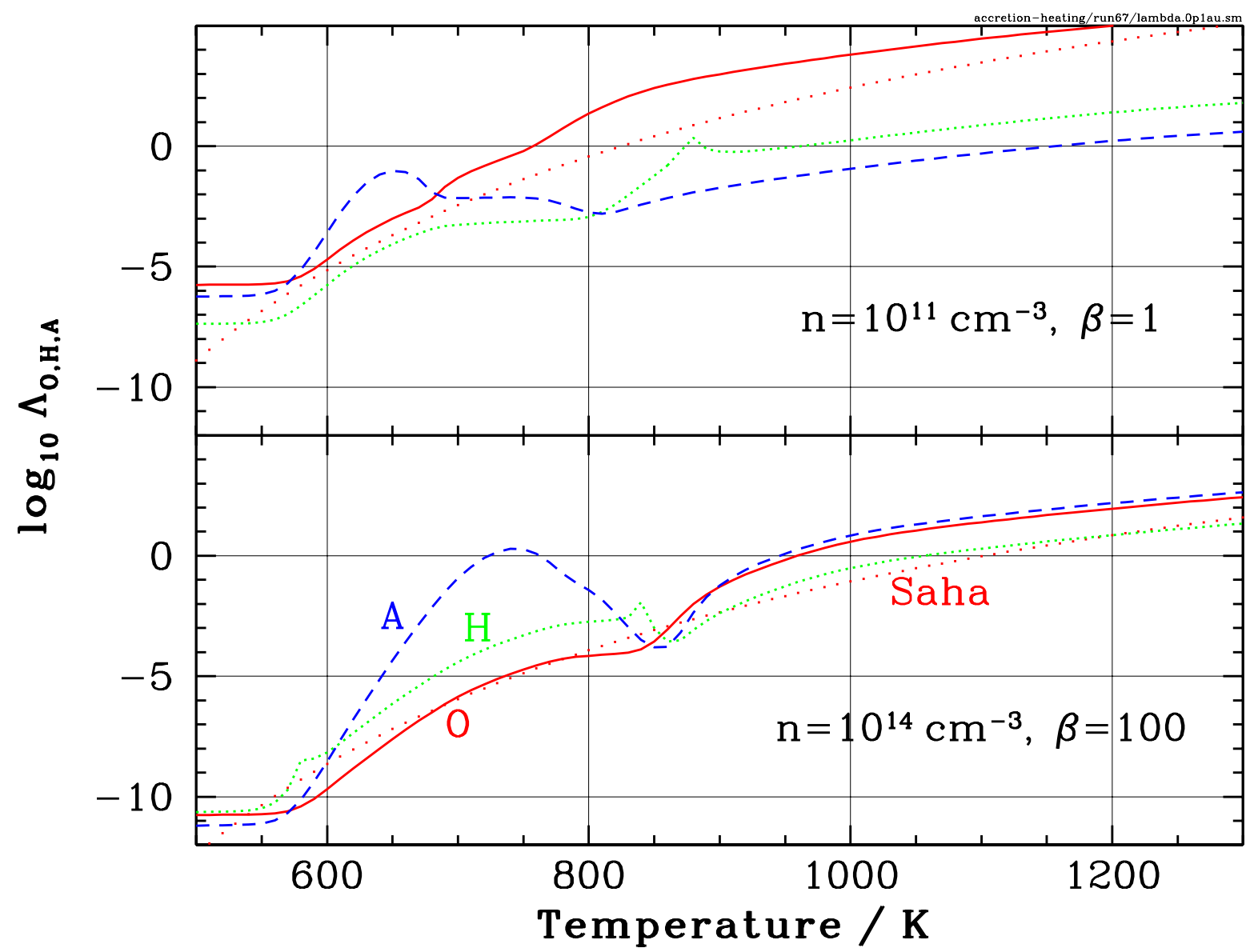

Fig. 17.- Ohmic Elsasser number $\Lambda_{\mathrm{O}}$ (red solid curve), ambipolar Elsasser number $\Lambda_{\mathrm{A}}$ (blue dashed curve), and Hall Elsasser number $\Lambda_{\mathrm{H}}$ (green dotted curve) as functions of temperature at $0.1 \mathrm{AU}$ from a Solar-mass star. The red dotted curve shows the Ohmic Elsasser number computed from the Saha equation for potassium, neglecting thermionic and ion emission. Different values of the density and plasma beta parameter in the top and bottom panels (top: $n_{\mathrm{H} 2}=10^{11} \mathrm{~cm}^{-3}, \beta=1$; (bottom: $n_{\mathrm{H} 2}=10^{14} \mathrm{~cm}^{-3}, \beta=100$ ) correspond to the disk atmosphere and midplane, respectively. 


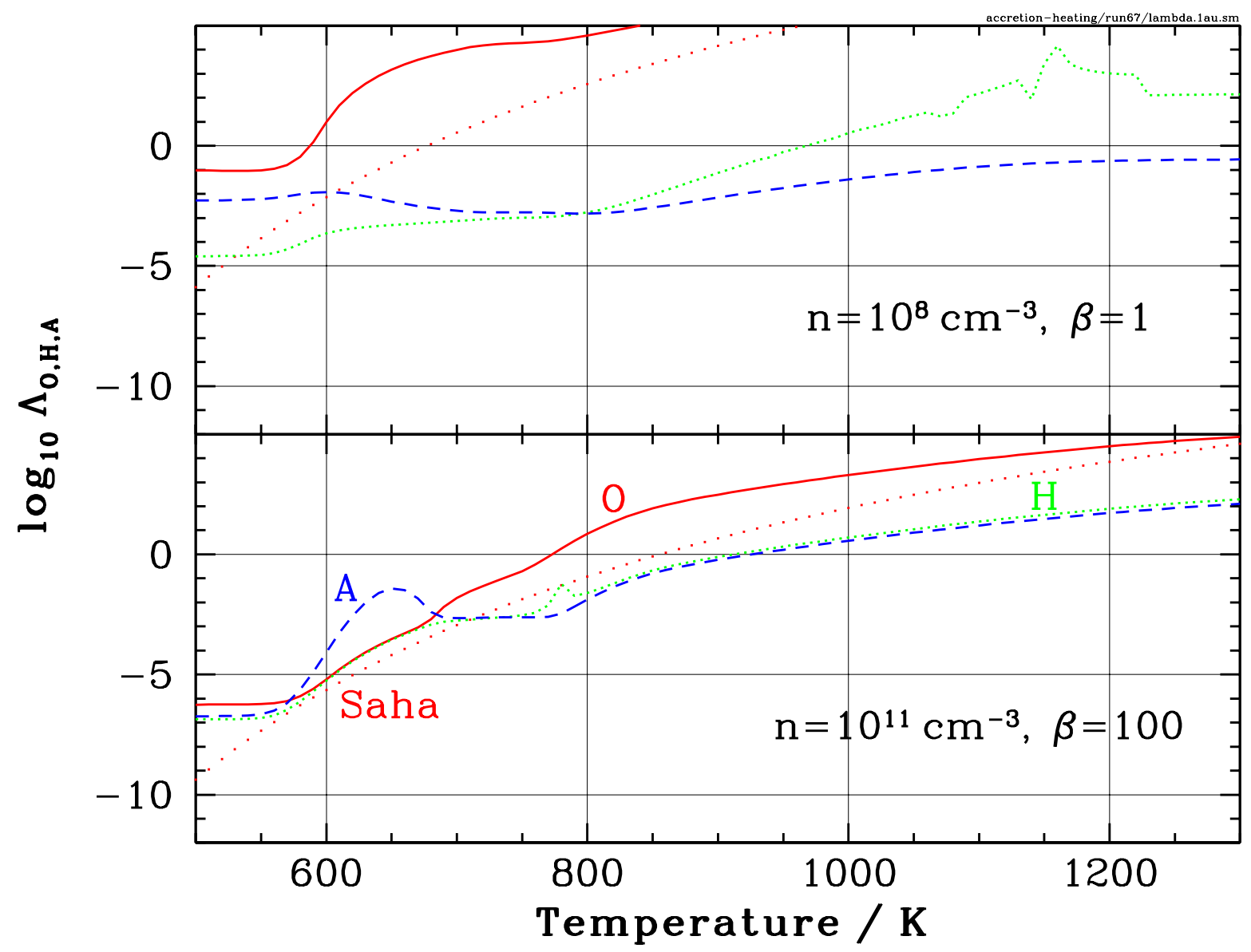

Fig. 18. - Same as Figure 17, but for heliocentric distance 1 AU. Ohmic Elsasser number $\Lambda_{\mathrm{O}}$ (red solid curve), ambipolar Elsasser number $\Lambda_{\mathrm{A}}$ (blue dashed curve), and Hall Elsasser number $\Lambda_{\mathrm{H}}$ (green dotted curve) are plotted vs. temperature $T$. The red dotted curve shows the Ohmic Elsasser number computed from the Saha equation for potassium. The top and bottom panels correspond to the disk atmosphere and midplane, respectively (top: $n_{\mathrm{H} 2}=10^{8} \mathrm{~cm}^{-3}, \beta=1$; bottom: $\left.n_{\mathrm{H} 2}=10^{11} \mathrm{~cm}^{-3}, \beta=100\right)$. 


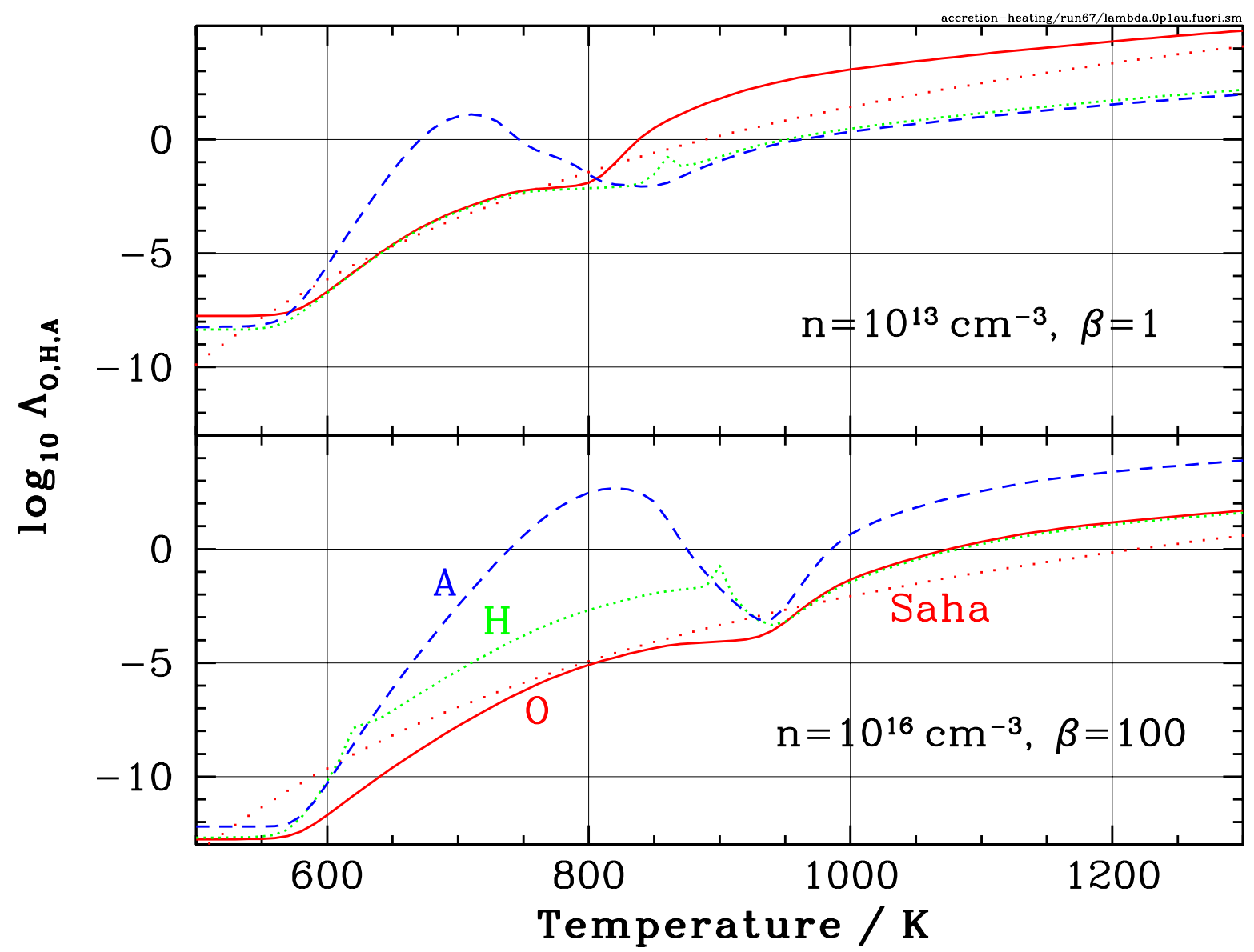

Fig. 19. - Same as Figure 17, except that the densities and plasma beta parameters correspond to the disk atmosphere and midplane at the onset of an accretion outburst, when gas and dust have piled up near $0.1 \mathrm{AU}$ (top panel: $n_{\mathrm{H} 2}=10^{13} \mathrm{~cm}^{-3}, \beta=1$; bottom: $n_{\mathrm{H} 2}=10^{16} \mathrm{~cm}^{-3}, \beta=100$ ). Ohmic Elsasser number $\Lambda_{\mathrm{O}}$ (red solid curve), ambipolar Elsasser number $\Lambda_{\mathrm{A}}$ (blue dashed curve), and Hall Elsasser number $\Lambda_{\mathrm{H}}$ (green dotted curve) are plotted vs. temperature $T$. The red dotted curve shows the Ohmic Elsasser number computed from the Saha equation for potassium. 


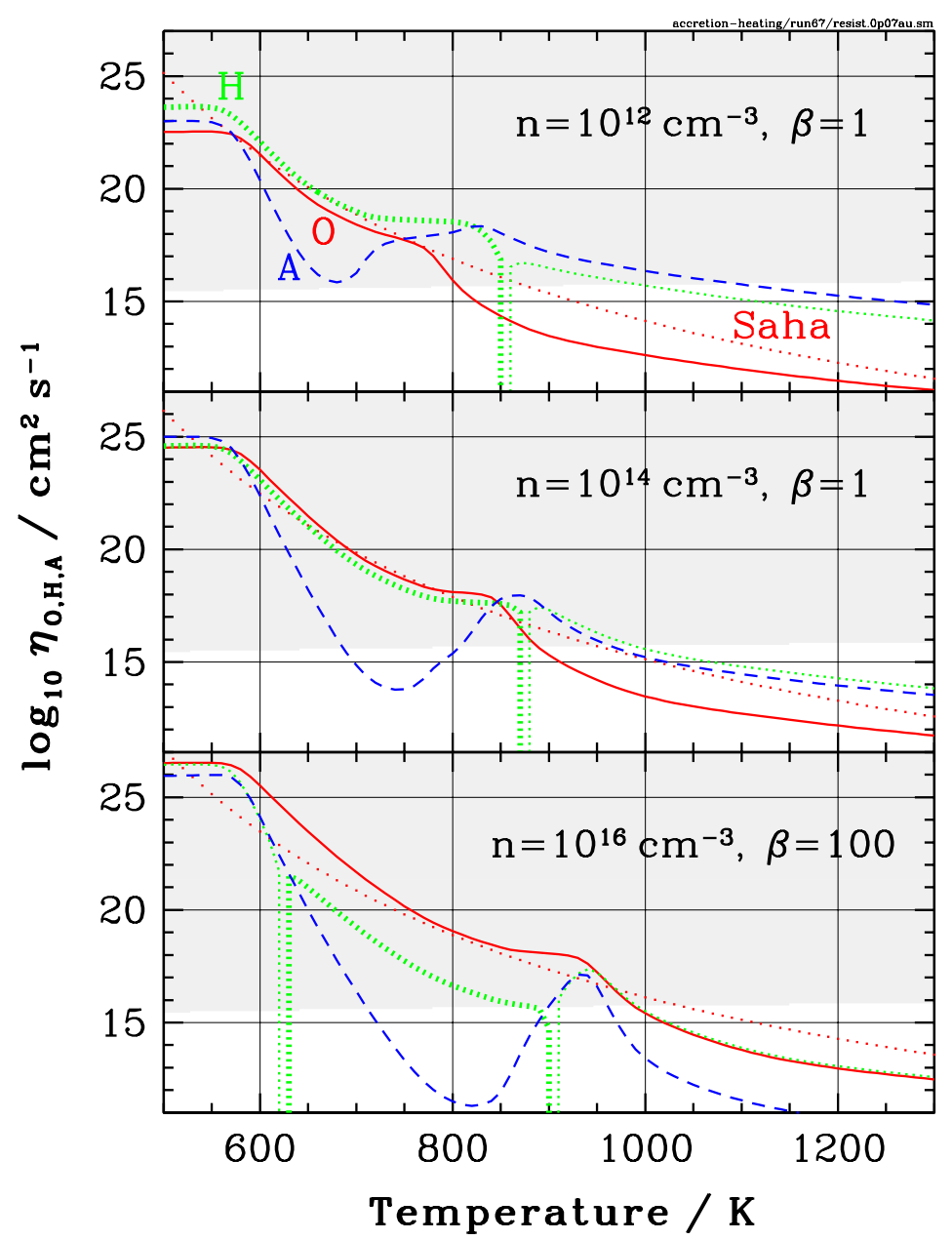

Fig. 20.- Magnetic diffusivities vs. temperature $T$, for fiducial grain parameters and three different combinations of gas density and plasma beta parameter. In each panel we plot the Ohmic (solid red curve), ambipolar (dashed blue curve), and Hall diffusivities (dotted green curve; the Hall diffusivity is negative on the thicker portion). Electron and ion abundances are derived using our chemical network including thermionic emission. Also plotted (red dotted curve) is the Ohmic diffusivity derived from the Saha equation for potassium. Gray shading indicates diffusivities greater than $h^{2} \Omega$, high enough that disk gas ought to be loaded onto the stellar fields primarily by diffusion. The threshold temperature is about $1000 \mathrm{~K}$ and is set by ambipolar diffusion except in the highest density case. 


\section{REFERENCES}

Ashton, A. F., \& Hayhurst, A. N. 1973, Combustion and Flame 21, 69.

Asplund, M., Grevesse, N., \& Sauval, A. J. 2005, Cosmic Abundances as Records of Stellar Evolution and Nucleosynthesis, 336, 25

Bai, X.-N., \& Goodman, J. 2009, ApJ, 701, 737

Bai, X.-N., \& Stone, J. M. 2011, ApJ, 736, 144

Bai, X.-N., \& Stone, J. M. 2013, ApJ, 769, 76

Balbus, S. A., \& Terquem, C. 2001, ApJ, 552, 235

Batygin, K., \& Stevenson, D. J. 2010, ApJ, 714, L238

Blaes, O. M., \& Balbus, S. A. 1994, ApJ, 421, 163

Bouvier, J., Cabrit, S., Fernandez, M., Martin, E. L., \& Matthews, J. M. 1993, A\&A, 272, 176

Bouvier, J., Alencar, S. H. P., Harries, T. J., Johns-Krull, C. M., \& Romanova, M. M. 2007, Protostars and Planets V, 479

Cleeves, L. I., Adams, F. C., \& Bergin, E. A. 2013a, ApJ, 772, 5

Cleeves, L. I., Adams, F. C., Bergin, E. A., \& Visser, R. 2013b, ApJ, 777, 28

Connolly, H. C., Jr., Desch, S. J., Ash, R. D., \& Jones, R. H. 2006, Meteorites and the Early Solar System II, 383

Crowell, C. R. 1965, Solid State Electronics, 8, 395

Desch, S. J. 1998, Ph.D. Thesis, University of Illinois, Urbana-Champaign 
Desch, S. J. 2004, ApJ, 608, 509

Desch, S. J. 2007, ApJ, 671, 878

Desch, S. J., \& Connolly, H. C., Jr. 2002, Meteoritics and Planetary Science, 37, 183

Desch, S. J., \& Cuzzi, J. N. 2000, Icarus, 143, 87

Draine, B. T., \& Sutin, B. 1987, ApJ, 320, 803

Dzyurkevich, N., Turner, N. J., Henning, T., \& Kley, W. 2013, ApJ, 765, 114

Edwards, S., Hartigan, P., Ghandour, L., \& Andrulis, C. 1994, AJ, 108, 1056

Ercolano, B., \& Glassgold, A. E. 2013, MNRAS, 436, 3446

Feuerbacher, B., Anderegg, M., Fitton, B., et al. 1972, Lunar and Planetary Science Conference Proceedings, 3, 2655

Fomenko, V. S. 1966, Handbook of Thermionic Properties, Plenum Press Data Division, 151 pp.

Fortney, J. J., Shabram, M., Showman, A. P., et al. 2010, ApJ, 709, 1396

Gammie, C. F. 1996, ApJ, 457, 355

Glassgold, A. E., Najita, J., \& Igea, J. 1997, ApJ, 480, 344

Glassgold, A. E., Najita, J., \& Igea, J. 2004, ApJ, 615, 972

Greiner, M. T., Chai, L., Helander, M. G., Tang, W.-M., \& Zheng-Hong, L. (2012) Advanced Functional Materials 22, 4557.

Hagstrom, M., Engvall, K., \& Pettersson, J. B. C. (2000) J. Phys. Chem. B 104, 4457.

Hasegawa, Y., \& Pudritz, R. E. 2010, MNRAS, 401, 143 
Hayashi, C. 1981, Progress of Theoretical Physics Supplement, 70, 35

Hirose, S., Uchida, Y., Shibata, K., \& Matsumoto, R. 1997, PASJ, 49, 193

Hirose, S., Krolik, J. H., \& Stone, J. M. 2006, ApJ, 640, 901

Hubbard, A., McNally, C. P., \& Mac Low, M.-M. 2012, ApJ, 761, 58

Igea, J., \& Glassgold, A. E. 1999, ApJ, 518, 848

Jain, S. C., \& Krishnan, K. S. 1952, Proc. Roy. Soc. London 213, 143.

Jin, L. 1996, ApJ, 457, 798

Kippenhahn, R., \& Weigert, A. 1990, Stellar Structure and Evolution, XVI, 468 pp. 192 figs.. Springer-Verlag Berlin Heidelberg New York. Also Astronomy and Astrophysics Library,

Kunz, M. W., \& Lesur, G. 2013, MNRAS, 434, 2295

Lesniak, M. V., \& Desch, S. J. 2011, ApJ, 740, 118

Lesur, G., Kunz, M. W., \& Fromang, S. 2014, A\&A, 566, 56

Lodders, K. 2003, ApJ, 591, 1220

MacPherson, G. J., Davis, A. M., \& Zinner, E. K. 1995, Meteoritics, 30, 365

McKay, D. S., Carter, J. L., Boles, W. W., Allen, C. C., \& Allton, J. H. 1993, Lunar and Planetary Science Conference, 24, 963

McNally, C. P., Hubbard, A., Mac Low, M.-M., Ebel, D. S., \& D’Alessio, P. 2013, ApJ, 767, L2

Menou, K. 2012, ApJ, 754, LL9 
Mihalas, D., \& Mihalas, B. W. 1984, New York, Oxford University Press, 1984, 731 p.,

Miller, K. A., \& Stone, J. M. 1997, ApJ, 489, 890

Miller, K. A., \& Stone, J. M. 2000, ApJ, 534, 398

Morris, M. A., \& Desch, S. J. 2010, ApJ, 722, 1474

Nakano, T., \& Umebayashi, T. 1986, MNRAS, 218, 663

Okuzumi, S. 2009, ApJ, 698, 1122

Oppenheimer, M., \& Dalgarno, A. 1974, ApJ, 192, 29

Perez-Becker, D., \& Chiang, E. 2011, ApJ, 735, 8

Perna, R., Menou, K., \& Rauscher, E. 2010, ApJ, 724, 313

Pneuman, G. W., \& Mitchell, T. P. 1965, Icarus, 4, 494

Rogers, T. M., \& Komacek, T. D. 2014, ApJ, 794, 132

Romanova, M. M., Kulkarni, A. K., \& Lovelace, R. V. E. 2008, ApJ, 673, L171

Sano, T., Miyama, S. M., Umebayashi, T., \& Nakano, T. 2000, ApJ, 543, 486

Sano, T., \& Inutsuka, S. 2001, ApJ, 561, 179

Sano, T. 2007, Ap\&SS, 307, 191

Scott, E. R. D., \& Krot, A. N. 2005, Chondrites and the Protoplanetary Disk, 341, 15

Trigwell, S., Captain, J. G., Arens, E. E., Quinn, J. W., \& Calle, C. I. (2009), IEEE Trans. Ind. Apps. 45, 1060.

Turner, N. J., Sano, T., \& Dziourkevitch, N. 2007, ApJ, 659, 729 
Umebayashi, T., \& Nakano, T. 1981, PASJ, 33, 617

Umebayashi, T., \& Nakano, T. 2009, ApJ, 690, 69

Wardle, M. 2007, Ap\&SS, 311, 35

Weidenschilling, S. J. 1977, Ap\&SS, 51, 153

Zhu, Z., Hartmann, L., Gammie, C. F., Book, L. G., Simon, J. B., \& Engelhard, E. 2010, ApJ, 713, 1143 